\title{
Particle Velocity Assisted Three Dimensional Sound Field Reproduction Using a Modal-Domain Approach
}

\author{
Huanyu Zuo, Student Member, IEEE, Thushara D. Abhayapala, Senior Member, IEEE \\ and Prasanga N. Samarasinghe, Member, IEEE
}

\begin{abstract}
In literature, particle velocity has been introduced to improve performance of spatial sound field reproduction systems. However, all existing work requires to have accurate particle velocity measurements at all of the discrete control points, which is difficult to obtain in real-world applications. In this work, we formulate continuous particle velocity expressions over space as a function of pressure coefficients in the modal domain that can be easily extracted by using a higher order microphone. The sound field within a target region is controlled by a weighted cost function we built to optimize the continuous particle velocity, as well as sound pressure, on the boundary of the region. In contrast to the conventional spatial sound field reproduction methods in the modal domain, the proposed method allows for non-uniform loudspeaker geometry with a limited number of loudspeakers, thus providing a flexible array arrangement. The performance of the proposed method is evaluated through numerical simulations in both a free field and a reverberant room. Finally, we prove the proposed method in an objective experiment with real-world measurements of room impulse response.
\end{abstract}

Index Terms-Particle velocity, spatial sound field reproduction, spherical harmonics, non-uniform loudspeaker array

\section{INTRODUCTION}

$\mathbf{S}$ PATIAL sound field reproduction is a fundamental technique in acoustics widely used in three dimensional (3D) audio systems, which aims to convincingly recreate a sound field in $3 \mathrm{D}$ space so that the listener experiences the sound field as a realistic but virtual replication of the original sound field. It is achieved by deriving the driving signals applied to the loudspeakers that encloses the spatial region of interest. In this paper, we propose a practical and accurate 3D sound field reproduction system that derives the driving signals with the aid of particle velocity in the modal domain.

Until now, there have been various studies on spatial sound field reproduction. Wave Field Synthesis (WFS) is a typical example initially conceived by Berkhout [1], [2], which synthesizes the desired sound field in a target region based on the Huygens-Fresnel principle by individually driven loudspeakers. Theoretically, it requires a continuous distribution of monopole and normally oriented dipole secondary sources on the boundary of the region [3]. An approximation to the continuous distribution is implemented by an array of

The authors are with the Research School of Engineering, College of Engineering and Computer Science, The Australian National University, Canberra, ACT 2601, Australia (e-mail: huanyu.zuo@anu.edu.au; prasanga.samarasinghe@anu.edu.au; thushara.abhayapala@anu.edu.au).

This work is supported by ARC Discovery Grant No. DP180102375 and the China Scholarship Council with the Australian National University. equally placed loudspeakers, and the reproduction artifacts due to the approximation is analyzed in [4]. To facilitate the implementation of WFS in a real reproduction environment, an adaptive WFS algorithm is proposed in [5]. Two dimensional WFS using linear and planar arrays is thoroughly studied in [6]-[9]. A practical implementation is called 2.5D WFS, which controls 2D sound fields using point sources serving as the secondary sources instead of line sources [10], [11]. However, there is a limited number of work implementing WFS in 3D space due to the fact that a significant number of loudspeakers are required for broadband reproduction over a large region [12]-[14]. Sound field reproduction can also be implemented by controlling sound pressure at a set of discrete sampling points, which is basically based on the least squares method [15], [16]. To improve the performance of the reproduction system in a large area, other optimization techniques are exploited, such as the wave field reconstruction filter [17], the singular value decomposition based optimization [18], and the least-absolute shrinkage and selection operator [19], [20].

Higher order ambisonics (HOA) is another approach to spatial sound field reproduction [21]-[25], which is achieved by extending Ambisonics that is based on the first order cylindrical harmonic analysis of the sound field [26]. HOA exploits higher order spherical harmonics to increase the spatial resolution, which is also widely used in multi-zone reproduction systems [27]-[29]. However, there is a requirement to place loudspeakers uniformly on a sphere that surrounds the target region so that the desired sound field within the region can be reconstructed perfectly [30], [31]. To facilitate the implementation of spherical arrays, multiple circular arrays have been proposed in HOA [32]-[34]. However, the performance of HOA deteriorates when the geometry of loudspeaker array becomes neither spherical nor circular due to the poorly conditioned matrix inversion. An improved least squares method with a weighted penalty function was developed for such irregular loudspeaker layouts [35]. This method requires the regularization parameter to be dependent on each loudspeaker arrangement. Ueno et. al. proposed a weighted mode matching method, which is flexible with various loudspeaker geometries, to avoid a relatively large effort devoted to matching the insignificant modes [36]. This method was also exploited for multizone reproduction [37], [38]. Sparse methods based HOA was investigated in [39], [40], which results in a significantly reduced number of the required microphones for the measuring process. In addition to the problem in terms of loudspeaker 
placement, there is another limitation for HOA. For a given order of a reproduction system, which is determined by the maximum frequency of the desired sound and the radius of the target region, HOA requires sufficient loudspeakers to match all the spherical harmonics to the given order in order to avoid spatial aliasing [21].

To overcome the limitations addressed above, particle velocity has been controlled in the sound field reproduction systems. Shin et al. proposed a particle velocity controlled sound field reproduction technique to simplify the regularization of the inverse problem for a non-uniformly spaced loudspeaker array [41], [42]. The work only considers particle velocity without sound pressure. A joint optimization, based on both sound pressure and particle velocity, was presented for broadband multi-zone sound field reproduction in [?], [43], where how the particle velocity impacts on the reproduction performance was explicitly analyzed. This work is restricted to reproduce the desired sound field in a 2D plane. Besides, all of these previous particle velocity based sound field reproduction methods focus on controlling the particle velocity at multiple discrete points on the boundary of the target region. To guarantee the accuracy of reproduced sound field over a large region, it requires a large quantity of control points, which implies that a practical system implementation requires to measure the particle velocity point by point for all the control points. The process of this measurement is time-consuming and costly.

The goal of this work is to incorporate particle velocity into HOA to investigate a flexible scheme for 3D sound field reproduction that can relax the limitations of loudspeaker number and placement. We derive continuous particle velocity in the modal domain (i.e., spherical harmonic domain) to avoid the process of measuring particle velocity at multiple discrete control points. The remainder of this paper is structured as follows. In Section II, the representations of continuous particle velocity are derived with infinite sets of closed form coefficients, and also finite sets of coefficients are suggested for practical implementation. The theory of velocity assisted $3 \mathrm{D}$ sound field reproduction is explicitly investigated for free fields and reverberant rooms in Section III. Section IV validates the proposed method by comparing it with conventional HOA (i.e., mode matching) in the aforementioned two environments. In Section V, we validate the method with an objective experiment using impulse response measurements of a real-world room. Finally, the work is concluded in Section VI.

\section{Continuous Particle Velocity in the Modal DOMAIN}

In this section, we represent particle velocity in the modal domain, and then decompose it in terms of spherical harmonics. We also derive closed form velocity coefficients and discuss velocity truncation error, allowing for continuous particle velocity to be exploited in the velocity-assisted sound field reproduction algorithm.

\section{A. Particle velocity vector}

Consider a source-free spherical region with radius $R$. The sound pressure at $\boldsymbol{x}=(r, \theta, \phi)$ in the spherical region, resulting from sources outside the region, is given by

$$
P(\boldsymbol{x}, k)=\sum_{n=0}^{N} \sum_{m=-n}^{n} \alpha_{n m}(k) j_{n}(k r) Y_{n m}(\theta, \phi)
$$

where $N=\lceil k e R / 2\rceil$ is the truncation order [44], $\alpha_{n m}(k)$ are modal-domain coefficients which describe the sound field incident to $\boldsymbol{x}$ due to the sources, $j_{n}(\cdot)$ is the $n^{\text {th }}$ order spherical Bessel function of the first kind, $k=2 \pi f / c$ is the wave number, $f$ is frequency, $c$ is the speed of sound propagation, and $Y_{n m}(\theta, \phi)$ is the spherical harmonic of order $n$ and degree $m$, defined by

$$
Y_{n m}(\theta, \phi) \triangleq \underbrace{\sqrt{\frac{(2 n+1)}{4 \pi} \frac{(n-m) !}{(n+m) !}}}_{A_{n m}} P_{n m}(\cos \theta) e^{i m \phi},
$$

where $P_{n m}(\cos \theta)$ is the associated Legendre function. The particle velocity vector $\boldsymbol{V}(\boldsymbol{x}, k)$ is related to the sound pressure at $\boldsymbol{x}$ by the Euler's equation [45],

$$
\boldsymbol{V}(\boldsymbol{x}, k)=\frac{i}{k \rho_{0} c} \vec{\nabla} P(\boldsymbol{x}, k),
$$

where $\rho_{0}$ is the medium density, $\boldsymbol{V}(\boldsymbol{x}, k)=V_{r}(\boldsymbol{x}, k) \hat{r}+$ $V_{\theta}(\boldsymbol{x}, k) \hat{\theta}+V_{\phi}(\boldsymbol{x}, k) \hat{\phi}$, and

$$
\vec{\nabla} \equiv \frac{\partial}{\partial r} \hat{r}+\frac{1}{r} \frac{\partial}{\partial \theta} \hat{\theta}+\frac{1}{r \sin \theta} \frac{\partial}{\partial \phi} \hat{\phi},
$$

where $\hat{r}, \hat{\theta}$, and $\hat{\phi}$ are the unit vectors in the $r, \theta$, and $\phi$ directions, respectively. Therefore, by substituting (1) into (3), we have

$$
\begin{aligned}
& V_{r}(\boldsymbol{x}, k)=\frac{i}{k \rho_{0} c} \sum_{n=0}^{N} \sum_{m=-n}^{n} \alpha_{n m}(k) j_{n}^{\prime}(k r) Y_{n m}(\theta, \phi) \\
& V_{\theta}(\boldsymbol{x}, k)= \\
& \frac{i}{k \rho_{0} c} \sum_{n=0}^{N} \sum_{m=-n}^{n} A_{n m} \alpha_{n m}(k) \frac{j_{n}(k r)}{r} P_{n m}^{\prime}(\cos \theta) e^{i m \phi}, \\
& V_{\phi}(\boldsymbol{x}, k)= \\
& -\frac{1}{k \rho_{0} c} \sum_{n=0}^{N} \sum_{m=-n}^{n} m A_{n m} \alpha_{n m}(k) \frac{j_{n}(k r)}{r} \frac{P_{n m}(\cos \theta)}{\sin \theta} e^{i m \phi} .
\end{aligned}
$$

where $j_{n}^{\prime}(k r)$ and $P_{n m}^{\prime}(\cos \theta)$ denote the derivative of $j_{n}(k r)$ in terms of $r$ and $P_{n m}(\cos \theta)$ in terms of $\theta$, respectively, expressed as

$$
j_{n}^{\prime}(k r)=\frac{n k j_{n-1}(k r)-(n+1) k j_{n+1}(k r)}{2 n+1},
$$

and

$$
\begin{aligned}
P_{n m}^{\prime}(\cos \theta) & =(n-m+1) \frac{P_{(n+1) m}(\cos \theta)}{\sin \theta} \\
& -(n+1) \frac{\cos \theta P_{n m}(\cos \theta)}{\sin \theta} .
\end{aligned}
$$




\section{B. Velocity coefficients}

Similar to sound pressure in (1), particle velocity can also be represented as a linear combination of basis functions, i.e., spherical harmonics in the modal domain. Therefore, (5), (6), and (7) can be decomposed as

$V_{\Psi}(\boldsymbol{x}, k)=\sum_{p=0}^{\infty} \sum_{q=-p}^{p} X_{p q}^{(\Psi)}(k, r) Y_{p q}(\theta, \phi) ; \quad \Psi=\{r, \theta, \phi\}$,

where $X_{p q}^{(\Psi)}(k, r)$ denote velocity coefficients in the $\Psi$ direction. The velocity coefficients in the $r$ direction can be obtained from (5) directly as

$$
X_{p q}^{(r)}(k, r)=\frac{i}{k \rho_{0} c} \alpha_{p q}(k) j_{p}^{\prime}(k r) .
$$

Derivations for the velocity coefficients in the $\theta$ and $\phi$ direction are given in Appendix $\mathrm{A}$. We introduce the results in the following theorem.

Theorem 1: The velocity coefficients in the $\theta$ direction $X_{p q}^{(\theta)}(k, r)$ and $\phi$ direction $X_{p q}^{(\phi)}(k, r)$ can be expressed, respectively, as

$$
\begin{gathered}
X_{p q}^{(\theta)}(k, r)=\frac{2 \pi i}{k \rho_{0} c} H_{p q} \sum_{n=|q|}^{N} H_{n q} \alpha_{n q}(k) \frac{j_{n}(k r)}{r} G_{1}, \\
X_{p q}^{(\phi)}(k, r)=-\frac{2 \pi q}{k \rho_{0} c} H_{p q} \sum_{n=|q|}^{N} H_{n q} \alpha_{n q}(k) \frac{j_{n}(k r)}{r} G_{2},
\end{gathered}
$$

where

$$
\begin{gathered}
H_{p q}=\frac{(-1)^{\frac{q+|q|}{2}}}{2^{|q|}(|q|) !} \sqrt{\frac{(2 p+1)}{4 \pi} \frac{(p+|q|) !}{(p-|q|) !}}, \\
G_{1}=(n+|q|+1) \mathcal{G}\left(a, b+\frac{1}{2}-\delta_{n+|q|+1} ;-\mu_{1}-\delta_{n+|q|+1},\right. \\
\left.-\mu_{2} ; \nu_{1}+\delta_{n+|q|}, \nu_{2} ; \xi_{1}, \xi_{2}\right)-(n+1) \mathcal{G}\left(a, b+\frac{1}{2} ;-\mu_{1},-\mu_{2} ;\right. \\
\left.\nu_{1}, \nu_{2} ; \xi_{1}, \xi_{2}\right),
\end{gathered}
$$

and

$$
\begin{aligned}
G_{2} & =\mathcal{G}\left(a, b ;-\mu_{1},-\mu_{2} ; \nu_{1}, \nu_{2} ; \xi_{1}, \xi_{2}\right) \\
& =\left[(-1)^{2 b+1}+1\right] \sum_{j_{1}=0}^{\mu_{1}} \sum_{j_{2}=0}^{\mu_{2}} \frac{\left(-\mu_{1}\right)_{j_{1}}\left(\nu_{1}\right)_{j_{1}}}{\left(\xi_{1}\right)_{j_{1}} j_{1} !} \frac{\left(-\mu_{2}\right)_{j_{2}}\left(\nu_{2}\right)_{j_{2}}}{\left(\xi_{2}\right)_{j_{2}} j_{2} !} \\
& \times \frac{B\left(j_{1}+j_{2}+a, b\right)}{2},
\end{aligned}
$$

$$
\begin{aligned}
& \text { show that given the spatial pressure field coefficients, spatial } \\
& \text { velocity coefficients can be directly calculated. }
\end{aligned}
$$

absolute value is more than $N$. From the above derivations we

\section{Velocity truncation error}

The representation (10) has an infinite number of orthogonal modes. To facilitate the implementation, we can truncate this decomposition to a finite number at a cost of a truncation error. For the particle velocity in the $\theta$ direction and $\phi$ direction, the infinite summations can be truncated to $Q_{\theta}$ and $Q_{\phi}$, respectively, as

$$
\begin{aligned}
& \hat{V}_{\theta}(\boldsymbol{x}, k)=\sum_{p=0}^{Q_{\theta}} \sum_{q=-p}^{p} X_{p q}^{(\theta)}(k, r) Y_{p q}(\theta, \phi), \\
& \hat{V}_{\phi}(\boldsymbol{x}, k)=\sum_{p=0}^{Q_{\phi}} \sum_{q=-p}^{p} X_{p q}^{(\phi)}(k, r) Y_{p q}(\theta, \phi),
\end{aligned}
$$

where $\hat{V}_{\theta}(\boldsymbol{x}, k)$ and $\hat{V}_{\phi}(\boldsymbol{x}, k)$ denote truncated particle velocity in the $\theta$ and $\phi$ direction, respectively. However, for the particle velocity in the $r$ direction, the representation with finite modes can be written directly by rearranging (5) as

$$
V_{r}(\boldsymbol{x}, k)=\sum_{p=0}^{Q_{r}} \sum_{q=-p}^{p} X_{p q}^{(r)}(k, r) Y_{p q}(\theta, \phi)
$$
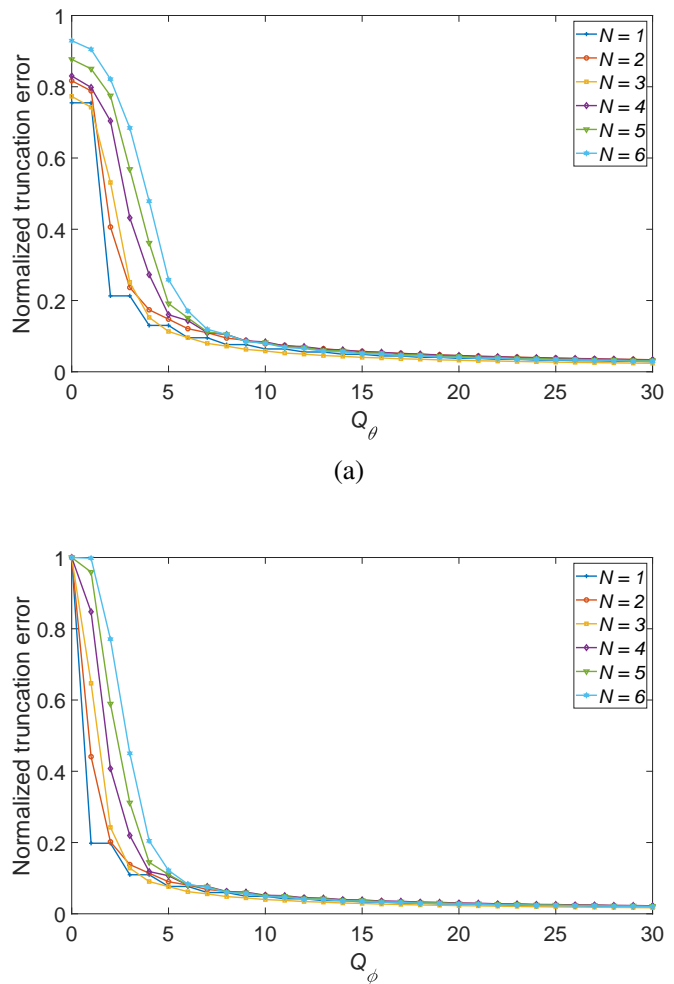

(b) Fig. 1. The normalized truncation error with respect to velocity truncation
order for (a) velocity in the $\theta$ direction and (b) velocity in the $\phi$ direction with various pressure truncation orders $N$.

$$
(a)_{j}= \begin{cases}1, & \text { if } j=0 \\ a(a+1) \ldots(a+j-1), & \text { if } j=1,2, \ldots,\end{cases}
$$

and $a=(2|q|+1) / 2, b=\left(3-\delta_{n+|q|}-\delta_{p+|q|}\right) / 2, \mu_{1}=$ $-\left(1-n+|q|-\delta_{n+|q|}\right) / 2, \mu_{2}=-\left(1-p+|q|-\delta_{p+|q|}\right) / 2$, $\nu_{1}=\left(2+n+|q|-\delta_{n+|q|}\right) / 2, \nu_{2}=\left(2+p+|q|-\delta_{p+|q|}\right) / 2$, $\xi_{1}=\xi_{2}=|q|+1$, and $B(\cdot)$ denotes the beta function. We note that both $X_{p q}^{(\theta)}(k, r)$ and $X_{p q}^{(\phi)}(k, r)$ are zero for any $q$ whose

with

$$
\delta_{M}= \begin{cases}1, & \text { if } M \text { is even } \\ 0, & \text { if } M \text { is odd }\end{cases}
$$




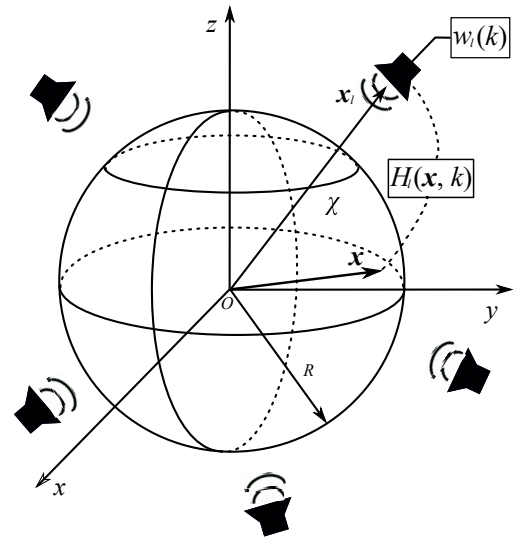

Fig. 2. Geometry of soundfield reproduction system in this paper. An array of loudspeakers is located outside $\chi$ with driving signals $w_{\ell}(k)$ and acoustic transfer function $H_{\ell}(\boldsymbol{x}, k)$ from the $\ell^{\text {th }}$ loudspeaker to a point $\boldsymbol{x}$ within the region.

where $Q_{r}=N$. To investigate the relationship between the truncation error and the truncation order, we define the normalized truncation error as

$$
\varepsilon_{\widetilde{\Psi}}(k)=\frac{\sum_{\forall \boldsymbol{x}}\left|V_{\widetilde{\Psi}}(\boldsymbol{x}, k)-\hat{V}_{\widetilde{\Psi}}(\boldsymbol{x}, k)\right|^{2}}{\sum_{\forall \boldsymbol{x}}\left|V_{\widetilde{\Psi}}(\boldsymbol{x}, k)\right|^{2}} ; \quad \widetilde{\Psi}=\{\theta, \phi\} .
$$

where $V_{\widetilde{\Psi}}(\boldsymbol{x}, k)$ is non-truncated particle velocity, which can be obtained from (6) and (7), and $\hat{V}_{\widetilde{\Psi}}(\boldsymbol{x}, k)$ is truncated particle velocity, which is measured by (19) and (20). Given a pressure truncation order, $N$, which are determined by the radius of the region of interest and the frequency as indicated in Section II-A, the normalized truncation error with respect to the velocity truncation order is shown in Fig. 1. We observe that the normalized truncation error decreases as the velocity truncation order increases, and falls to a small value rapidly for all the cases, which means the lower modes contain the majority of the energy and therefore we can truncate the infinite summations to a particular order with a tolerant error. Note that the higher the truncation order is, the less the truncation error becomes but the greater computational complexity the system has.

\section{VELOCITY ASSISTED SOUND FIELD REPRODUCTION}

The theory of velocity-assisted sound field reproduction algorithm is presented in this section. Before the driving signals of loudspeakers are designed by jointly optimizing the sound pressure and the particle velocity in Section III-C, we model the point-to-region transfer function between each loudspeaker to the target region for both a free field and a reverberant environment in Section III-B. Finally, 3D sound field reproduction error is given in Section III-D.

\section{A. Problem definition}

Our objective is to reproduce the pressure $P_{d}(\boldsymbol{x}, k)$ of a desired sound field at any point $\boldsymbol{x}$ within a source free spherical region $\chi$ of radius $R$ as shown in Fig. 2. The desired sound field can be a plane wave, a point source or a field measured in a real-world scenario. Let there be an array of loudspeakers outside of $\chi$, with the $\ell^{\text {th }}$ loudspeaker located at $\boldsymbol{x}_{\ell}=\left(r_{\ell}, \theta_{\ell}, \phi_{\ell}\right)$ with respect to the origin $O$. The sound pressure at $\boldsymbol{x}$ due to the $\ell^{\text {th }}$ loudspeaker can be written as

$$
P_{\ell}(\boldsymbol{x}, k)=w_{\ell} H_{\ell}(\boldsymbol{x}, k),
$$

where $w_{\ell}$ is the driving signal applying to the $\ell^{\text {th }}$ loudspeaker, and $H_{\ell}(\boldsymbol{x}, k)$ is the acoustic transfer function between the $\ell^{\text {th }}$ loudspeaker to $\boldsymbol{x}$ for arbitrary environments. Assume there is a total of $L$ loudspeakers, therefore, the total reproduced sound pressure at $\boldsymbol{x}$ is given by

$$
P_{a}(\boldsymbol{x}, k)=\sum_{\ell=1}^{L} w_{\ell} H_{\ell}(\boldsymbol{x}, k) .
$$

The design task of sound field reproduction is to find the loudspeaker driving signals that can reproduce the desired sound field optimally. The popular approach to solving this problem is to minimize the least squares error between the desired sound field and the reproduced sound field within the region $\chi$, i.e.,

$$
\min _{\boldsymbol{x} \in \chi}\left\|P_{d}(\boldsymbol{x}, k)-P_{a}(\boldsymbol{x}, k)\right\|_{2}^{2},
$$

where $\|\cdot\|_{2}$ denotes the Euclidean norm. This can be formulated as mode matching [21] by expressing $P_{d}(\boldsymbol{x}, k)$ and $P_{a}(\boldsymbol{x}, k)$ in terms of spherical harmonics similar to (1) in the modal domain. Although a 3D sound field can be accurately reproduced by this method, it requires at least $(N+1)^{2}$ loudspeakers uniformly distributed on a sphere. The performance of mode matching degrades when there is only a limited number of loudspeakers or the loudspeaker geometry is irregular. It is the constraint we wish to relax through the introduction of particle velocity vectors, which we present next.

\section{B. Point-to-region transfer function}

Acoustic transfer function plays an important role in sound field reproduction because it reflects how much sound from a source is observed by a receiver. The acoustic transfer function between the $\ell^{\text {th }}$ loudspeaker to $\boldsymbol{x}, H_{\ell}(\boldsymbol{x}, k)$, can be expressed by the modal decomposition as

$$
H_{\ell}(\boldsymbol{x}, k)=\sum_{n=0}^{N} \sum_{m=-n}^{n} \beta_{n m}^{(\ell)}(k) j_{n}(k r) Y_{n m}(\theta, \phi),
$$

where $\beta_{n m}^{(\ell)}(k)$ are the sound field coefficients of the acoustic transfer function. We also refer to (26) as the point-toregion transfer function, because the acoustic transfer function between the $\ell^{\text {th }}$ loudspeaker to any point in the region $\chi$ is readily available if $\beta_{n m}^{(\ell)}(k)$ are known. The coefficients $\beta_{n m}^{(\ell)}(k)$ can also represent the directional characteristics of the loudspeaker [46], therefore, (26) can be the acoustic transfer function due to not only omni-directional sources but also directional loudspeakers. For simplicity, in the following two sections, we assume all loudspeakers to be omni-directional. Note that in practice the aforementioned point-region transfer function coefficients related to a given loudspeaker can be 
extracted from recordings of higher order microphones such as an EigenMike [47], by feeding a sweep signal into the loudspeaker.

1) Free field: In a free field, the point-to-region transfer function $H_{\ell}^{\mathrm{dir}}(\boldsymbol{x}, k)$ only contains the direct path component, which is determined by the Green's function [45],

$$
\begin{aligned}
H_{\ell}^{\mathrm{dir}}(\boldsymbol{x}, k) & =\frac{e^{i k\left\|\boldsymbol{x}-\boldsymbol{x}_{\ell}\right\|_{2}}}{4 \pi\left\|\boldsymbol{x}-\boldsymbol{x}_{\ell}\right\|_{2}} \\
= & \sum_{n=0}^{N} \sum_{m=-n}^{n} i k h_{n}\left(k r_{\ell}\right) Y_{n m}^{*}\left(\theta_{\ell}, \phi_{\ell}\right) j_{n}(k r) Y_{n m}(\theta, \phi),
\end{aligned}
$$

where $h_{n}(\cdot)$ is the $n^{\text {th }}$ order spherical Hankel function of the first kind and $*$ denotes complex conjugate. In this case, the coefficients of the point-to-region transfer function due to the $\ell^{\text {th }}$ loudspeaker can be expressed as

$$
\beta_{n m}^{(\ell)}(k)=i k h_{n}\left(k r_{\ell}\right) Y_{n m}^{*}\left(\theta_{\ell}, \phi_{\ell}\right)
$$

2) Reverberant environment: We model the reverberant environment as a shoebox room, and derive the point-toregion transfer function using the spherical harmonics based generalized image source method [46]. The point-to-region transfer function in the reverberant environment due to the $\ell^{\text {th }}$ loudspeaker $H_{\ell}^{\mathrm{rvb}}(\boldsymbol{x}, k)$ can be expressed as

$$
H_{\ell}^{\mathrm{rvb}}(\boldsymbol{x}, k)=\sum_{n=0}^{N} \sum_{m=-n}^{n} \frac{i k}{\sqrt{4 \pi}} \alpha_{n m}^{00}(k) j_{n}(k r) Y_{n m}(\theta, \phi) \text {, }
$$

where $\alpha_{n m}^{00}(k)$ denotes the coupling coefficients. The exact expression of $\alpha_{n m}^{00}(k)$, as well as the proof of (29), is given in Appendix B. Therefore, in the reverberant environment, the expression of the point-to-region transfer function coefficients due to the $\ell^{\text {th }}$ loudspeaker is

$$
\beta_{n m}^{(\ell)}(k)=\frac{i k}{\sqrt{4 \pi}} \alpha_{n m}^{00}(k),
$$

where the location information of the $\ell^{\text {th }}$ loudspeaker is incorporated in $\alpha_{n m}^{00}(k)$. Note that (30) holds only for the simulated shoebox room. For a real-world room with arbitrary geometries, one can estimate the coefficients from impulse response measurements, which we present in Section V.

\section{Velocity assisted optimization}

The proposed method is based on the Kirchhoff-Helmholtz integral equation, which states that the sound pressure is completely determined within a volume free of sources, if sound pressure and particle velocity are controlled in all points on its surface. Therefore, the 3D sound field reproduction problem is reduced to optimizing the sound pressure and particle velocity on the surface of the region $\chi$. Different from controlling multiple discrete points on the surface [43], we exploit the continuous sound pressure and particle velocity on the surface in the modal domain. From (1), the desired sound pressure can be given by

$$
P_{d}(\boldsymbol{x}, k)=\sum_{n=0}^{N} \sum_{m=-n}^{n} \underbrace{\alpha_{n m}^{(d)}(k) j_{n}(k r)}_{\hat{\alpha}_{n m}^{(d)}(k, r)} Y_{n m}(\theta, \phi),
$$

where $\alpha_{n m}^{(d)}(k)$ are the pressure coefficients of the desired sound field. Replacing $\alpha_{n m}(k)$ with $\alpha_{n m}^{(d)}(k)$ in (11), (12), and (13) and substituting them into (10), we have the desired particle velocity as

$$
V_{\Psi}^{(d)}(\boldsymbol{x}, k)=\sum_{p=0}^{Q_{\Psi}} \sum_{q=-p}^{p} X_{p q}^{(\Psi, d)}(k, r) Y_{p q}(\theta, \phi) .
$$

Similarly, by replacing $\alpha_{n m}(k)$ with $\beta_{n m}^{(\ell)}(k)$, the reproduced sound pressure and particle velocity due to the loudspeaker array can be written, respectively, as

$$
\begin{gathered}
P_{a}(\boldsymbol{x}, k)=\sum_{n=0}^{N} \sum_{m=-n}^{n} \underbrace{\sum_{\ell=1}^{L} w_{\ell} \beta_{n m}^{(\ell)}(k) j_{n}(k r)}_{\hat{\beta}_{n m}^{(a)}(k, r)} Y_{n m}(\theta, \phi), \\
V_{\Psi}^{(a)}(\boldsymbol{x}, k)=\sum_{p=0}^{Q_{\Psi}} \sum_{q=-p}^{p} \underbrace{\sum_{\ell=1}^{L} w_{\ell} X_{p q}^{(\Psi, \ell)}(k, r)}_{X_{p q}^{(\Psi, a)}(k, r)} Y_{p q}(\theta, \phi) .
\end{gathered}
$$

Note that $\hat{\alpha}_{n m}^{(d)}(k, r) / X_{p q}^{(\Psi, d)}(k, r)$ and $\hat{\beta}_{n m}^{(a)}(k, r) / X_{p q}^{(\Psi, a)}(k, r)$ are the desired and reproduced pressure/particle velocity coefficients on the surface with the radius of $r$, respectively, which represent the continuous sound pressure/particle velocity on the surface. Using these coefficients, a weighted least squares optimization criterion, including both sound pressure and particle velocity on the surface of $\chi$, can be formulated

$$
\begin{aligned}
\min _{\boldsymbol{W}}\left\{\tau^{2}\left\|\boldsymbol{\beta}_{A}(k, R) \boldsymbol{W}(k)-\boldsymbol{\alpha}_{D}(k, R)\right\|_{2}^{2}\right. \\
\left.\quad+(1-\tau)^{2}\left\|\boldsymbol{X}_{A}(k, R) \boldsymbol{W}(k)-\boldsymbol{X}_{D}(k, R)\right\|_{2}^{2}\right\},
\end{aligned}
$$

where $\tau \in[0,1]$ is the weighting coefficient to adjust the relative weight of sound pressure and particle velocity, $\boldsymbol{\alpha}_{D}(k, R)=$

$\left[\alpha_{00}^{(d)}(k) j_{0}(k R), \alpha_{1(-1)}^{(d)}(k) j_{1}(k R), \ldots, \alpha_{N N}^{(d)}(k) j_{N}(k R)\right]^{T}$ is a $(N+1)^{2}$ long vector,

$\boldsymbol{X}_{D}(k, R)=\left[X_{00}^{(r, d)}(k, R), \ldots, X_{Q_{r} Q_{r}}^{(r, d)}(k, R), X_{00}^{(\theta, d)}(k, R)\right.$, $\left.\ldots, X_{Q_{\theta} Q_{\theta}}^{(\theta, d)}(k, R), X_{00}^{(\phi, d)}(k, R), \ldots, X_{Q_{\phi} Q_{\phi}}^{(\phi, d)}(k, R)\right]^{T} \quad$ is $\quad$ a $\left[\left(Q_{r}+1\right)^{2}+\left(Q_{\theta}+1\right)^{2}+\left(Q_{\phi}+1\right)^{2}\right]$ long vector containing all three components of the particle velocity vector, $\left.\boldsymbol{W}(k)=\left[w_{1}(k), w_{2}(k), \ldots, w_{L}(k)\right)\right]^{T}$ is a $L$ long vector, $\boldsymbol{\beta}_{A}(k, R)$ is a $(N+1)^{2}$ by $L$ matrix given by

$$
\boldsymbol{\beta}_{A}(k, R)=\left[\begin{array}{ccc}
\beta_{00}^{(1)} j_{0}(k R) & \cdots & \beta_{00}^{(L)} j_{0}(k R) \\
\beta_{1(-1)}^{(1)} j_{1}(k R) & \cdots & \beta_{1(-1)}^{(L)} j_{1}(k R) \\
\vdots & \ddots & \vdots \\
\beta_{N N}^{(1)} j_{N}(k R) & \cdots & \beta_{N N}^{(L)} j_{N}(k R)
\end{array}\right],
$$

and $\boldsymbol{X}_{A}(k, R)=\left[\boldsymbol{X}_{r}(k, R)^{T}, \boldsymbol{X}_{\theta}(k, R)^{T}, \boldsymbol{X}_{\phi}(k, R)^{T}\right]^{T}$ is a $\left[\left(Q_{r}+1\right)^{2}+\left(Q_{\theta}+1\right)^{2}+\left(Q_{\phi}+1\right)^{2}\right]$ by $L$ matrix with

$$
\boldsymbol{X}_{\Psi}(k, R)=\left[\begin{array}{ccc}
X_{00}^{(\Psi, 1)}(k, R) & \cdots & X_{00}^{(\Psi, L)}(k, R) \\
X_{1(-1)}^{(\Psi, 1)}(k, R) & \cdots & X_{1(-1)}^{(\Psi, L)}(k, R) \\
\vdots & \ddots & \vdots \\
X_{Q_{\Psi}^{(\Psi, 1)}(k, R)}^{\left(Q_{\Psi}\right.}(k, \cdots & X_{Q_{\Psi} Q_{\Psi}}^{(\Psi, L)}(k, R)
\end{array}\right] .
$$


The optimization problem (35) can be rearranged using stacked matrices as

$$
\min _{\boldsymbol{W}}\left\|\hat{\boldsymbol{X}}_{A}(k, R) \boldsymbol{W}(k)-\hat{\boldsymbol{X}}_{D}(k, R)\right\|_{2}^{2}
$$

where $\hat{\boldsymbol{X}}_{A}(k, R)=\left[\tau \boldsymbol{\beta}_{A}(k, R)^{T},(1-\tau) \boldsymbol{X}_{A}(k, R)^{T}\right]^{T}$, and $\hat{\boldsymbol{X}}_{D}(k, R)=\left[\tau \boldsymbol{\alpha}_{D}(k, R)^{T},(1-\tau) \boldsymbol{X}_{D}(k, R)^{T}\right]^{T}$. This problem can be solved using a Moore-Penrose inverse with Tikhonov regularization [48]. We introduce the weighting coefficient $\tau$ to make the optimization criterion adjustable for different scenarios. Note that the pressure coefficients in (38) are weighted by the Bessel functions and therefore $\tau=1$ corresponds to the weighted mode matching by the Bessel functions rather than the conventional pressure matching in the modal domain. The Bessel functions in the optimization criterion can be replaced with other functions such as the Gaussian-weighted Bessel functions [38], and the proposed velocity-assisted method can also work.

\section{Reproduction error}

We define the normalized reproduction error over the spherical target region as

$$
\epsilon(k)=\sqrt{\frac{\int_{0}^{R} \int_{0}^{2 \pi} \int_{0}^{\pi}\left|P_{d}(\boldsymbol{x}, k)-P_{a}(\boldsymbol{x}, k)\right|^{2} d \Omega}{\int_{0}^{R} \int_{0}^{2 \pi} \int_{0}^{\pi}\left|P_{d}(\boldsymbol{x}, k)\right|^{2} d \Omega}}
$$

where $d \Omega=r^{2} \sin \theta d \theta d \phi d r$. Substituting (31) and (33) into (39), it can be simplified as

$$
\begin{aligned}
& \epsilon(k)= \\
& \sqrt{\frac{\sum_{n=0}^{N} \sum_{m=-n}^{n}\left|\alpha_{n m}^{(d)}(k)-\sum_{\ell=1}^{L} w_{l} \beta_{n m}^{(\ell)}(k)\right|^{2} \mathcal{J}_{n}(k, R)}{\sum_{n=0}^{N} \sum_{m=-n}^{n}\left|\alpha_{n m}^{(d)}(k)\right|^{2} \mathcal{J}_{n}(k, R)}},
\end{aligned}
$$

where

$$
\begin{aligned}
& \mathcal{J}_{n}(k, R)= \\
& \begin{cases}{[2 k R-\sin (2 k R)] / 4 k^{3},} & \text { if } n=0 \\
R^{3}\left[j_{n}^{2}(k R)-j_{n-1}(k R) j_{n+1}(k R)\right] / 2, & \text { if } n>0 .\end{cases}
\end{aligned}
$$

Equation (40) is a closed-form expression for 3D sound field reproduction error, and the proof of (40) is given in Appendix B.

\section{Simulations}

In this section, we illustrate the performance of the proposed method in various simulated environments using two different loudspeaker geometries. First, the analysis of determining the weighting coefficient for the reproduction system is given. Using this weighting coefficient, the system is implemented and compared with the conventional pressure matching in the modal domain (or mode matching) [21], [22], in terms of the reproduced sound pressure, virtual source position, frequency, and reproduced sound direction.
TABLE I

LOUDSPEAKER POSITIONS OF THE 22 CHANNEL SYSTEM

\begin{tabular}{cccc}
\hline \hline Loudspeaker No. & $r[\mathrm{~m}]$ & $\theta[\mathrm{deg}]$ & $\phi[\mathrm{deg}]$ \\
\hline 1 & 0.97 & 0 & 0 \\
2 & 1.55 & 51.3 & 0 \\
3 & 1.55 & 51.3 & 45 \\
4 & 1.55 & 51.3 & 90 \\
5 & 1.55 & 51.3 & 135 \\
6 & 1.55 & 51.3 & 180 \\
7 & 1.55 & 51.3 & 225 \\
8 & 1.55 & 51.3 & 270 \\
9 & 1.55 & 51.3 & 315 \\
10 & 1.21 & 90 & 0 \\
11 & 1.21 & 90 & 22.5 \\
12 & 1.21 & 90 & 45 \\
13 & 1.21 & 90 & 90 \\
14 & 1.21 & 90 & 135 \\
15 & 1.21 & 90 & 180 \\
16 & 1.21 & 90 & 225 \\
17 & 1.21 & 90 & 270 \\
18 & 1.21 & 90 & 315 \\
19 & 1.21 & 90 & 337.5 \\
20 & 1.34 & 115 & 0 \\
21 & 1.34 & 115 & 45 \\
22 & 1.34 & 115 & 315 \\
\hline \hline
\end{tabular}

\section{A. Simulation setup}

In this simulation example, the radius of the region $\chi$ is set to $R=0.18 \mathrm{~m}$. We simulate two different 3D loudspeaker layouts. One is a spherical array, where there are 25 loudspeakers uniformly placed on the surface of a $1 \mathrm{~m}$ sphere at locations determined by [49]. Another loudspeaker array is based on the cylindrical layout of 22.2 channel system proposed by NHK [50] without the two lowfrequency channels, where the loudspeaker position of the array is given in Table I. The loudspeakers are all modeled as ideal point sources. For the reverberant environments, we simulate a large room of size $(5,6,5) \mathrm{m}$ and a small room of size $(2.5,3,2.4) \mathrm{m}$, with the same reflection coefficients of $\boldsymbol{d}=[0.8,0.7,0.8,0.7,0.78,0.81]$. The reverberant time $T_{60}$ is $0.35 \mathrm{~s}$ for the large room and $0.17 \mathrm{~s}$ for the small room, respectively, calculated by the Sabine formula [51]. The image depth is $\boldsymbol{R}_{\text {depth }}=(4,4,4)$. A virtual point source with frequency of $1200 \mathrm{~Hz}$ at $(2, \pi / 9,2 \pi / 3)$ is the desired sound source throughout the simulations, except the evaluation in terms of the virtual source position and frequency. Sound speed is $c=343 \mathrm{~m} / \mathrm{s}$ and air density is $\rho_{0}=1.29 \mathrm{~kg} / \mathrm{m}^{3}$. Therefore, the pressure truncation order is $N=6$. The velocity truncation order is set to $Q_{\theta}=Q_{\phi}=2 N$.

The reproduction error over the whole spherical target region is calculate by (40) throughout the paper. To show the difference between the reproduced sound field and the desired sound field intuitively, we define the mismatch between them at any point $\boldsymbol{x}$ as

$$
\eta(\boldsymbol{x}, k)=\frac{\left|P_{d}(\boldsymbol{x}, k)-P_{a}(\boldsymbol{x}, k)\right|^{2}}{\left|P_{d}(\boldsymbol{x}, k)\right|^{2}} \times 100(\%) .
$$

From the perspective of realistic perception of source location, to reproduce the original sound, it requires to ensure the reproduction of the direction of the sound. A convenient measure for the direction of energy propagation in an arbitrary 


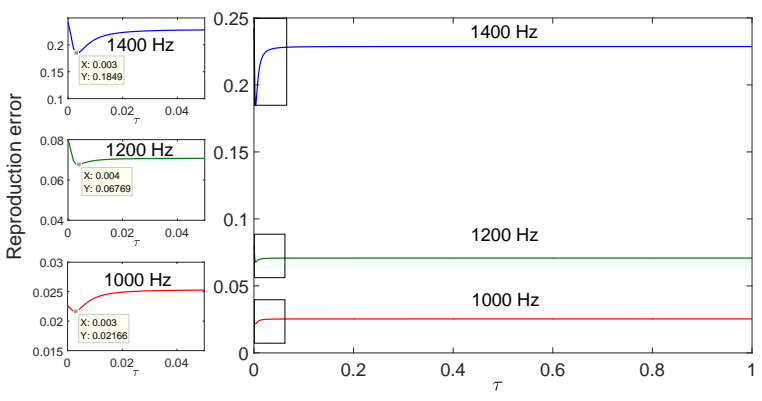

(a)

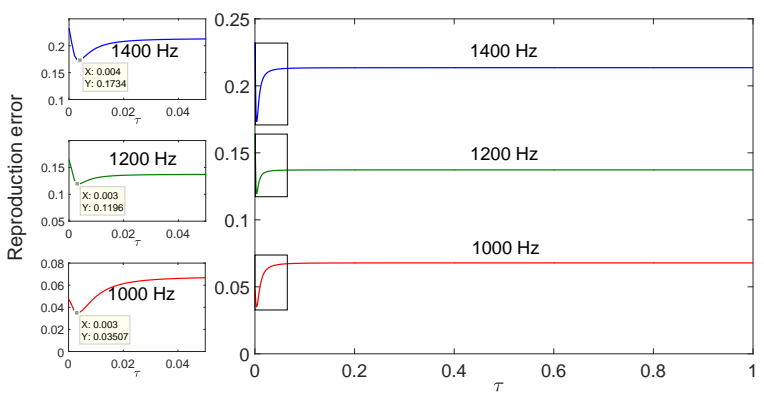

(b)

Fig. 3. The impact of the weighting coefficient on the reproduction system for various frequencies in (a) the free field and (b) the reverberant environment (the large room) in the 22 channel loudspeaker layout. The sub-figures on the left are the enlarged curves in the rectangular boxes correspondingly.

sound field is provided by the active sound intensity, which is defined for steady state fields as [45]

$$
\boldsymbol{I}(\boldsymbol{x}, k)=\frac{1}{2} \operatorname{Re}\left\{P^{*}(\boldsymbol{x}, k) \boldsymbol{V}(\boldsymbol{x}, k)\right\},
$$

where $\operatorname{Re}(\cdot)$ denotes the real part and * denotes complex conjugate. Note that the active sound intensity is a vector containing the information of direction, and it has been shown as a good predictor of localization perception [42], [52], [53]. To show the difference in angles between two vectors, we define the intensity direction error $\xi$ at $\boldsymbol{x}$ as

$$
\xi(\boldsymbol{x}, k)=\cos ^{-1}\left[\hat{\boldsymbol{I}}_{a}(\boldsymbol{x}, k) \cdot \hat{\boldsymbol{I}}_{d}(\boldsymbol{x}, k)\right] / \pi \times 100 \%,
$$

where $\hat{\boldsymbol{I}}_{a}(\boldsymbol{x}, k)=\boldsymbol{I}_{a}(\boldsymbol{x}, k) /\left|\boldsymbol{I}_{a}(\boldsymbol{x}, k)\right|$ and $\hat{\boldsymbol{I}}_{d}(\boldsymbol{x}, k)=$ $\boldsymbol{I}_{d}(\boldsymbol{x}, k) /\left|\boldsymbol{I}_{d}(\boldsymbol{x}, k)\right|$ are the unit active intensity vector of the reproduced sound field and the unit active intensity vector of the desired sound field, respectively.

\section{B. Determination of the weighting coefficient}

Before evaluating the overall performance of the proposed method, we first investigate how the weighting coefficient $\tau$ impacts the reproduction system. As shown in Section III-C, the weighting coefficient controls the relative weight of sound pressure and particle velocity. Note that the larger the weighting coefficient is, the less weight it assigns to the particle velocity. We calculate the 3D reproduction error with respect to $\tau$ for various frequencies in both the free field and the reverberant environment (the large room) using the 22 channel loudspeaker array, which is shown in Fig. 3. The curves in the rectangular boxes are enlarged and shown on the left correspondingly. We observe that the reproduction system has minimum error when $\tau$ is 0.003 approximately for all the evaluated frequencies in both the free field and the reverberant environment. For the values more than $0.1, \tau$ has little influence on the reproduction system, which means more weight should be assigned to the particle velocity to make it take effect because of the significant difference between the values of sound pressure and particle velocity. This result also means the velocity-assisted method with an appropriate weighting coefficient has better performance than the weighted mode matching by the Bessel functions (i.e., $\tau=1$ ). We also notice that the reproduction system has more error when the frequency increases, because the system order goes up. The optimization criterion with $\tau=0.003$ also works well for the 25 channel spherical array and the small room, therefore, we use the weighting coefficient $\tau=0.003$ for the reproduction system in the following analysis. Note that this value is optimal only for the simulation scenarios in this paper. One should recalculate the optimal weighting coefficient if the scenario totally changes because the difference between the values of pressure coefficients and velocity coefficients may also change, which may result in a different optimal value.

\section{Simulation results in free field}

The simulations in this section study the overall performance of the proposed method in free field for the two loudspeaker arrangements.

1) Reproduced sound pressure: We now evaluate the performance of the system on the reproduction of sound pressure. The observation plane is the plane of $z=0$. Figure 4 and Fig. 5 show the desired sound field and the reconstructed sound fields controlled by the pressure matching method and the velocity-assisted method in both loudspeaker layouts. The black circle denotes the target region. As expected, the velocity-assisted method provides a better sound field reproduction than the pressure matching method within the target region. The mismatches between the desired sound field and the reconstructed sound field for both methods are also shown in Fig. 4 and Fig. 5, which is calculated by (42). We observe that the pressure matching method fails to reproduce the desired sound field for the whole target region in the 22 channel system. When the loudspeaker array changes to the 25 channel regular spherical array, the performance of the pressure matching method has a significant improvement, however, it is still not as good as the velocity-assisted method. In this case, it requires at least $(N+1)^{2}=49$ uniformly distributed loudspeakers to accurately reproduce the desired sound field in the target region for the pressure matching method, whereas there are only 22 non-uniformly placed loudspeakers or 25 regular loudspeakers available. However, the velocity-assisted method reproduces the desired sound field in the target region with much less error than the pressure matching method verifying that the proposed method can overcome this limitation.

2) Virtual source position: To investigate the overall performance of the proposed method with respect to the virtual source position, we keep the distance $(2 \mathrm{~m})$ from the virtual 


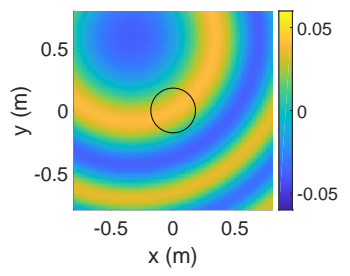

(a)

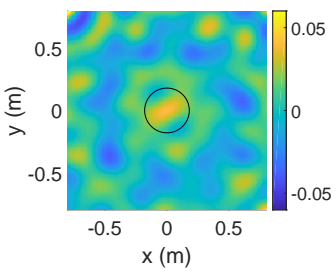

(b)

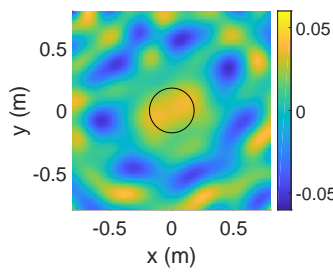

(c)

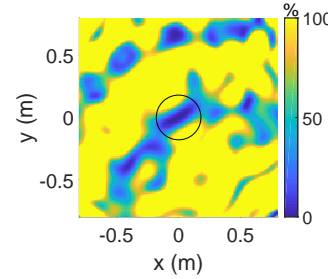

(d)

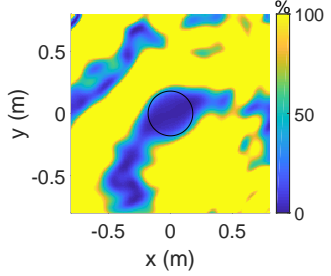

(e)

Fig. 4. Desired sound field, reconstructed sound field, and the difference between them on the observation plane controlled by both methods for the 22 channel system. (a) Desired sound field; (b) Reproduced sound field controlled by PMM; (c) Reproduced sound field controlled by VAM; (d) Difference field controlled by PMM; (e) Difference field controlled by VAM.

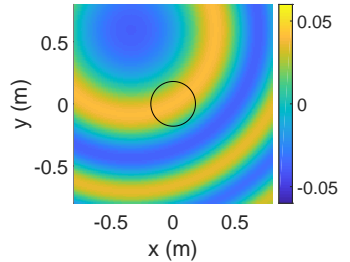

(a)

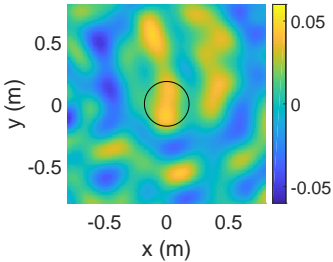

(b)

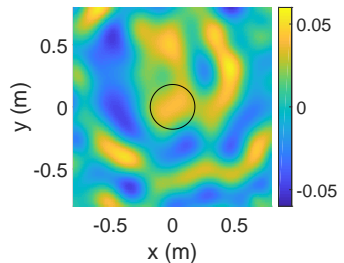

(c)

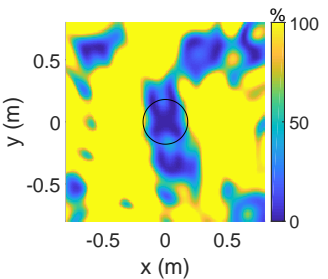

(d)

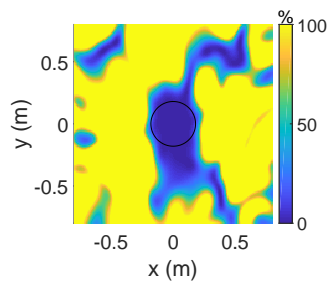

(e)

Fig. 5. Desired sound field, reconstructed sound field, and the difference between them on the observation plane controlled by both methods for the 25 channel system. (a) Desired sound field; (b) Reproduced sound field controlled by PMM; (c) Reproduced sound field controlled by VAM; (d) Difference field controlled by PMM; (e) Difference field controlled by VAM.

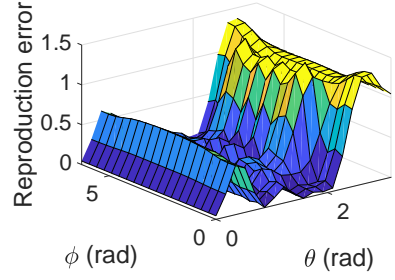

(a)

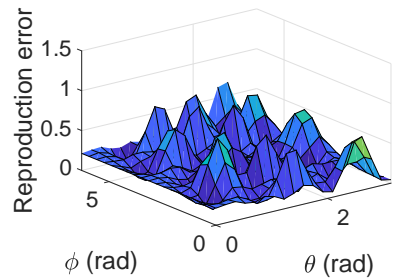

(c)

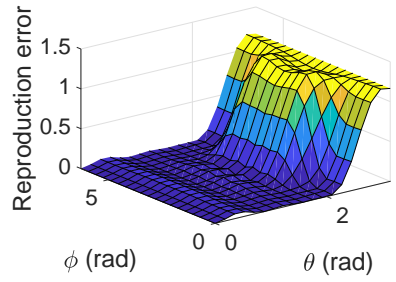

(b)

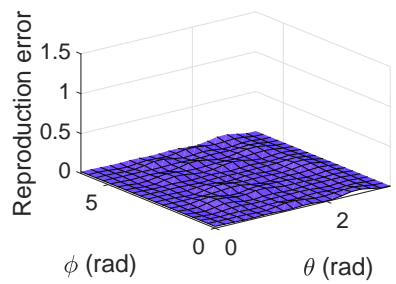

(d)
Fig. 6. 3D reproduction error with respect to the virtual source positions controlled by $(a, c)$ PMM and (b, d) VAM for $(a, b)$ the 22 channel system and $(\mathrm{c}, \mathrm{d})$ the 25 channel system.

source to the origin fixed and calculate the 3D reproduction error in terms of $\theta$ and $\phi$. The three-dimensional surface plots showing the results are given in Fig. 6. By comparing the error plots for the two loudspeaker arrays, we notice that the change of the reproduction error is regular with the virtual source position change for the 25 channel regular loudspeaker array, whereas for the 22 channel loudspeaker array the error fluctuates dramatically as the virtual source position changes due to the non-uniformly distributed loudspeakers. For the 22 channel irregular loudspeaker array, the velocity-assisted

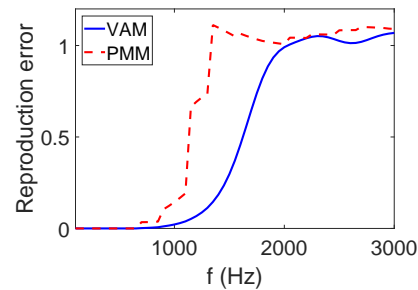

(a)

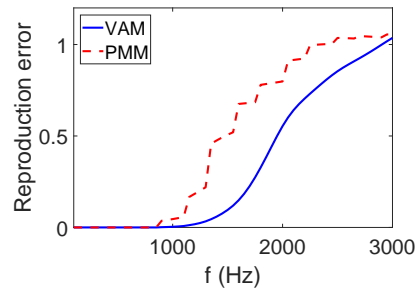

(b)
Fig. 7. 3D reproduction error with respect to the change of frequency controlled by both methods for (a) the 22 channel system and (b) the 25 channel system.

method performs better than the pressure matching method when the virtual source is located in the upper hemisphere $(0 \leq \theta \leq \pi / 2)$, however, the performance of both methods degrades for the virtual sources from the lower hemisphere $(\pi / 2<\theta \leq \pi)$ because there are only 3 out of 22 loudspeakers located below $x-y$ plane. In contrast, the 3D reproduction error is less than 0.1 for all the virtual source positions using the 25 channel regular loudspeaker array, which is much better than the pressure matching method.

3) Frequency: To analyze the broadband performance of the proposed method, we also calculate the 3D reproduction error with respect to the change of frequency from $100 \mathrm{~Hz}$ to $3000 \mathrm{~Hz}$, which is shown in Fig. 7. From Fig. 7, we observe that the error remains very low for both methods in the 22 channel system at frequencies less than $650 \mathrm{~Hz}$ approximately, where the system order $N$ is smaller than 3 . The 22 loudspeakers are enough to accurately reproduce the desired sound field in the target region for both methods. As the frequency increases ( $N$ also increases), the 22 loudspeakers are not enough 


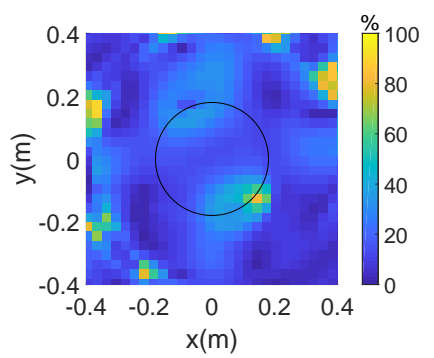

(a)

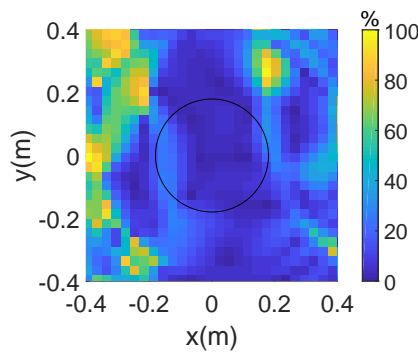

(c)

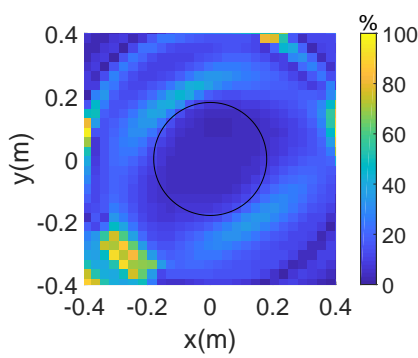

(b)

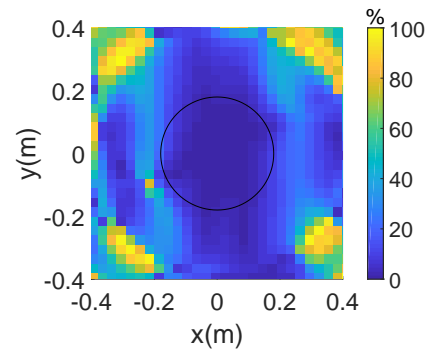

(d)
Fig. 8. The intensity direction error on plane $z=0$ controlled by (a, c) PMM and $(b, d)$ VAM for $(a, b)$ the 22 channel system and $(c, d)$ the 25 channel system.

any more and the error increases dramatically for the pressure matching method. However, the error builds up more gradually for the velocity-assisted method. For example, the error due to the pressure matching method is more than 1 , whereas the error due to the velocity-assisted method is only around 0.3 when the frequency increases to $1500 \mathrm{~Hz}$. Therefore, given a loudspeaker array and a target region, the velocity-assisted method can reproduce the desired sound field with a wider frequency range than the pressure matching method. We note that, when the frequency continues to increase to $3000 \mathrm{~Hz}$, the velocity-assisted method loses this advantage and its error also increases to around 1 because of the extreme scarcity of loudspeakers. The overall trend of error curve in the 25 channel system is same as that in the 22 channel system for both methods, however, the former is lower than the latter for all evaluated frequencies due to the increase of the number of loudspeakers.

4) Intensity direction: As we mentioned in Section IV-A, it is clear that if we are concerned to create a realistic perception of the original sound, it requires to ensure the reproduction of the direction of travel of the sound. Therefore, we calculate the intensity direction error on plane $z=0$ using (44), which is shown in Fig. 8. In the 22 channel system, the intensity direction error of the velocity-assisted method is less than $20 \%$ within the region of interest, whereas it is around $40 \%$ for the pressure matching method. When the loudspeaker array becomes regular, the intensity direction error of the pressure matching method reduces, however, it is still larger than the velocity-assisted method. This analysis shows that the velocity-assisted method can reproduce the direction of original sound inside the region of interest in free field.

From the above analysis, we conclude that the velocityassisted method has better overall performance than the pres-

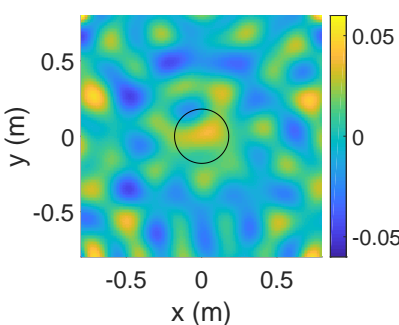

(a)

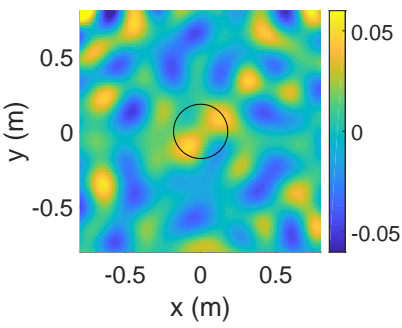

(c)

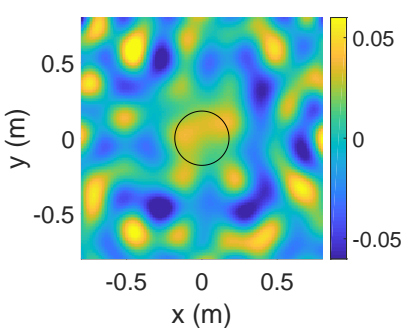

(b)

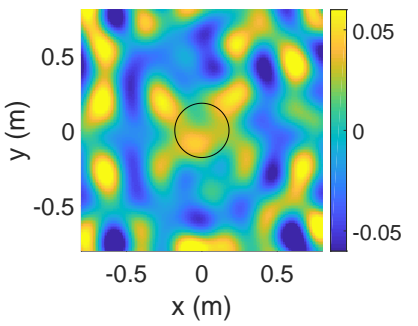

(d)
Fig. 9. Reconstructed sound field on the observation plane controlled by (a, c) PMM and (b, d) VAM in (a, b) the large room and (c, d) the small room in case of the 22 channel system.

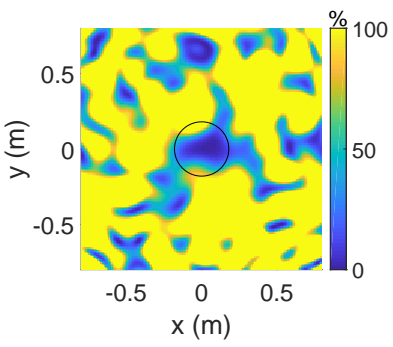

(a)

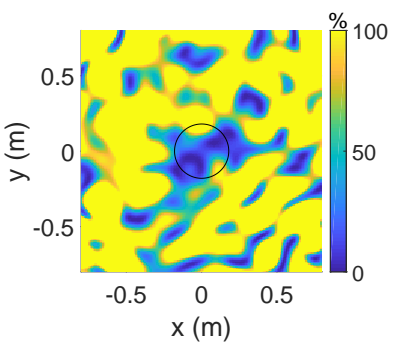

(c)

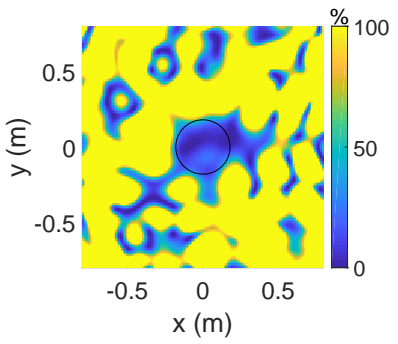

(b)

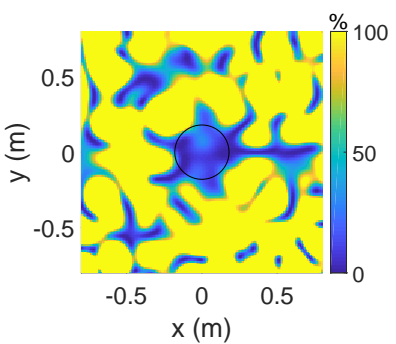

(d)
Fig. 10. Difference between the desired sound field and the reconstructed sound field controlled by $(a, c)$ PMM and (b, d) VAM in (a, b) the large room and (c, d) the small room in case of the 22 channel system.

sure matching method in free field, especially for a nonuniformly distributed loudspeaker array with a limited number of loudspeakers.

\section{Simulation results in reverberant rooms}

The following simulations investigate the overall performance of the proposed method in the two different rooms. Here we only consider the 22 channel non-uniformly distributed loudspeaker array. 


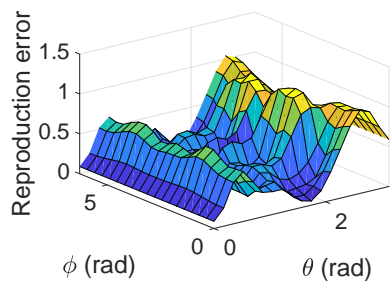

(a)

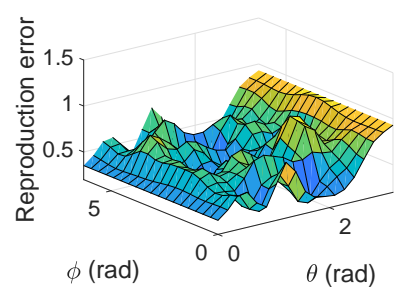

(c)

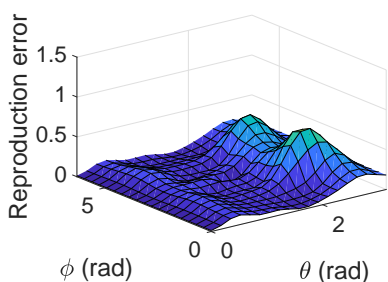

(b)

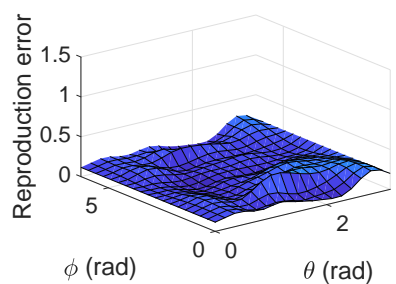

(d)
Fig. 11. 3D reproduction error with respect to the virtual source positions controlled by $(a, c)$ PMM and (b, d) VAM in $(a, b)$ the large room and (c, d) the small room in case of the 22 channel system.

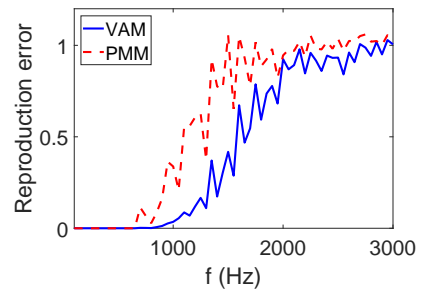

(a)

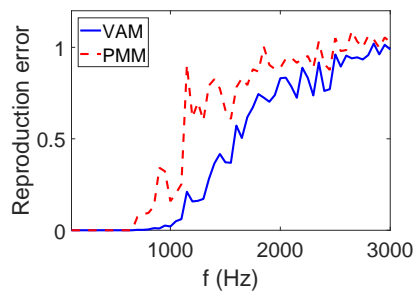

(b)
Fig. 12. 3D reproduction error with respect to the change of frequency controlled by both methods in (a) the large room and (b) the small room in case of the 22 channel system.

1) Reproduced sound pressure: We also first evaluate the reproduction of sound pressure within the region of interest, and the results in the different sizes of rooms are given in Fig. 9. The difference between the desired sound field and the reconstructed sound field is shown in Fig. 10. It shows that the velocity-assisted method provides more accurate pressure reproduction in both the reverberant rooms. By comparing with the results in the free field, we observe that for this particular virtual source the performance in the reverberant environment is a little worse than that in the free field. However, this result is not consistent for all the virtual source positions. The reproduction performance with respect to the position of the virtual source in the reverberant rooms is discussed next.

2) Virtual source position: The same virtual source positions as in Section IV-C are evaluated for the reverberant environments. The results are given in Fig. 11. By comparing the results in the free field and the reverberant environment, we notice that the performance in the free field is parallel to the performance in the reverberant environment for the virtual sources from the upper hemisphere, where there are more loudspeakers. However, for the virtual sources from

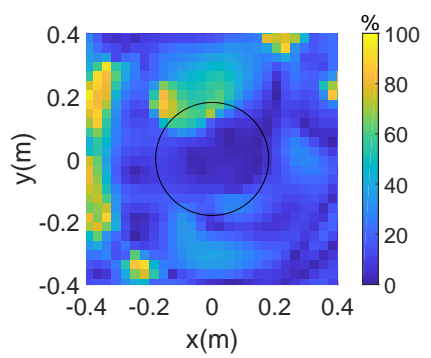

(a)

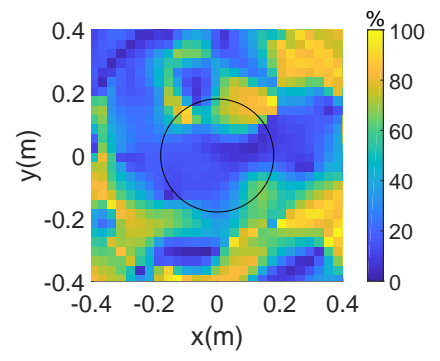

(c)

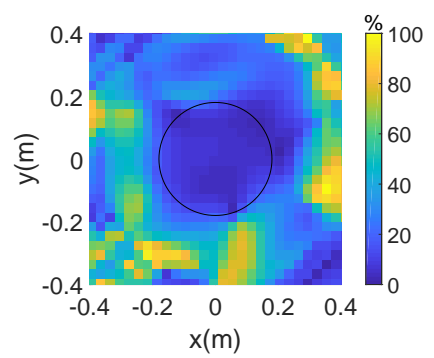

(b)

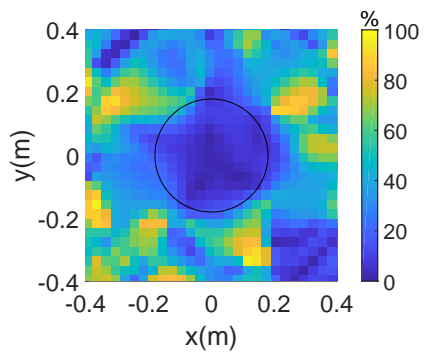

(d)
Fig. 13. The intensity direction error on plane $z=0$ controlled by (a, c) PMM and (b, d) VAM in (a, b) the large room and (c, d) the small room in case of the 22 channel system.

the lower hemisphere where there are few loudspeakers, the sound pressure reproduction in the reverberant environment has less error than that in the free field. This is because the reflections (image sources) in the reverberant room make up for the insufficiency of loudspeakers in the lower hemisphere in this case. Compared with the pressure matching method, the $3 \mathrm{D}$ reproduction error of the velocity-assisted method is less than 0.5 for all the virtual source positions in the reverberant environment, which is much better than the pressure matching method. We also note that the velocity-assisted method has more improvement from the remedy due to the reflections in the reverberant room than the pressure matching method. This is because not only sound pressure but also particle velocity is exploited for the reflections that are incorporated in the optimization criterion of the velocity-assisted method.

3) Frequency: Fig. 12 shows the results of the two methods with respect to the change of frequency in the two different sizes of rooms. The performance in the large room is similar to that in the small room for both methods. Similar to the results in the free field, the error starts to increase at the frequency of $650 \mathrm{~Hz}$, and reaches 1 at the frequency of $2000 \mathrm{~Hz}$. Therefore, the overall trend of error curve in the free field is same as that in the reverberant environment for both methods, however, the former is more smooth than the latter due to the more complicated environment in a reverberant room.

4) Intensity direction: The intensity direction error is also calculated for both methods in the reverberant rooms, which is given in Fig. 13. From Fig. 13, we show that the pressure matching method cannot guarantee accurate reproduction of sound direction inside the whole target region in the reverberant environment, whereas the intensity direction error of the velocity-assisted method is less than $20 \%$ for the whole target region. Although the performance of the pressure matching 


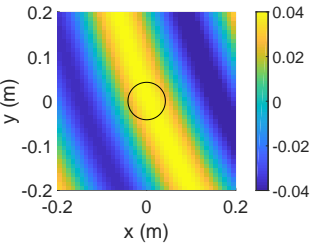

(a)

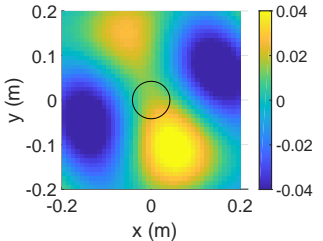

(b)

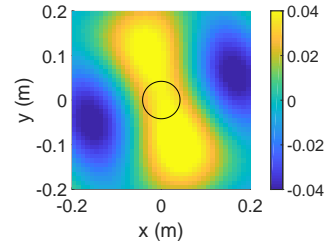

(c)

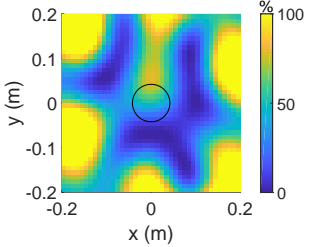

(d)

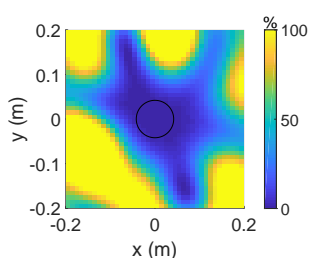

(e)

Fig. 14. Desired sound field, reconstructed sound field, and the difference between them on the observation plane controlled by both methods for source \#1. (a) Desired sound field; (b) Reproduced sound field controlled by PMM; (c) Reproduced sound field controlled by VAM; (d) Difference field controlled by PMM; (e) Difference field controlled by VAM.

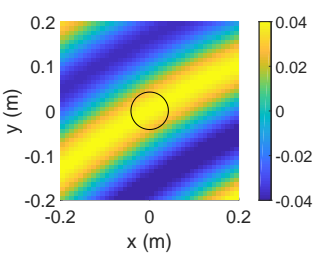

(a)

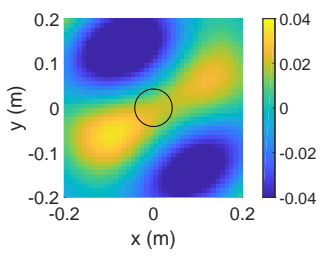

(b)

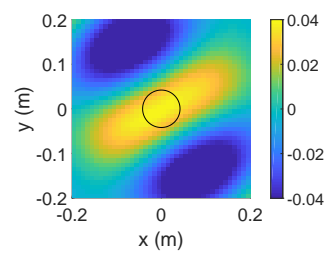

(c)

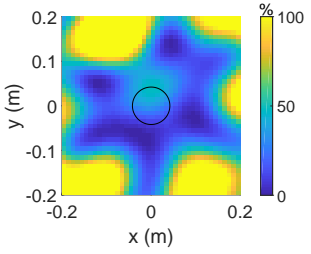

(d)

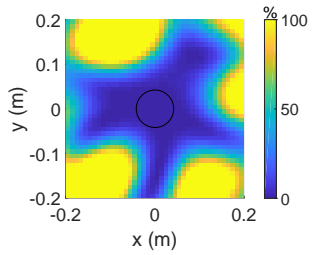

(e)

Fig. 15. Desired sound field, reconstructed sound field, and the difference between them on the observation plane controlled by both methods for source \#2. (a) Desired sound field; (b) Reproduced sound field controlled by PMM; (c) Reproduced sound field controlled by VAM; (d) Difference field controlled by PMM; (e) Difference field controlled by VAM.

method in the large room is slightly better than that in the small room, it is at the nearly same level for the performance of the velocity-assisted method in the two rooms. Therefore, we prove that the velocity-assisted method can reconstruct the original sound direction in various sizes of rooms. According to the above analysis, we conclude that the velocity-assisted method performs better than the pressure matching method in the reverberant environment for all the assessed aspects. The size of the reverberant room has little effect on the performance of the reproduction system.

The evaluation in this section has shown that the proposed method is better than the pressure matching method for overall performance in both free fields and reverberant environments. However, we should note that the computational complexity of the proposed method is higher than that of the pressure matching method due to the introduction of particle velocity.

\section{EXPERIMENTAL VERIFICATION}

In this section, we use the impulse response measurements in a real-world room to validate the proposed method. The impulse responses were measured in an office space at the Australian National University with dimensions of $(3.54,4.06,2.70) \mathrm{m}$. For simplicity, we consider a circular loudspeaker array with radius of $1 \mathrm{~m}$, consisting of six non-uniformly placed loudspeakers. We use such a circular loudspeaker array as it has a significant reduced implementation complexity compared to the loudspeaker geometries in Section IV. However, the implementation steps for the proposed method remain unchanged. We set the center of the circular array as the origin, and the loudspeakers are positioned on the horizontal plane $\left(\theta=90^{\circ}\right)$ with $\phi=48^{\circ}, 92^{\circ}$, $148^{\circ}, 184^{\circ}, 256^{\circ}$, and $336^{\circ}$, respectively. An EigenMike [47] located at the origin is used for sound field recording, where the EigenMike is a 32 channel rigid spherical microphone array with radius of $0.042 \mathrm{~m}$. A 2 second linear sweep signal is fed into each loudspeaker and the resulting sound field is recorded by the EigenMike. The impulse response between each loudspeaker and each EigenMike sensor can be obtained from the recordings, therefore we have $32 \times 6$ impulse responses measurements. The coefficients of the point-toregion transfer function between each loudspeaker and the receiver region (i.e., the EigenMike area) are estimated from the corresponding 32 channel measurements by

$$
\hat{\beta}_{n m}^{(\ell)}(k)=\frac{1}{b_{n}\left(k R_{E}\right)} \sum_{q_{M}=1}^{Q_{M}} \hat{H}_{\ell}\left(k, \boldsymbol{x}_{q_{M}}\right) Y_{n m}^{*}\left(\theta_{q_{M}}, \phi_{q_{M}}\right),
$$

where $\hat{H}_{\ell}\left(k, \boldsymbol{x}_{q_{M}}\right)$ is the measurement between the $\ell^{\text {th }}$ loudspeaker and the $q_{M}^{\text {th }}$ EigenMike sensor located at $\boldsymbol{x}_{q_{M}}=$ $\left(r_{q_{M}}, \theta_{q_{M}}, \phi_{q_{M}}\right), Q_{M}=32$ is the number of EigenMike sensors, $R_{E}=0.042 \mathrm{~m}$ is the radius of the EigenMike, and

$$
b_{n}\left(k R_{E}\right)=j_{n}\left(k R_{E}\right)-\frac{j_{n}^{\prime}\left(k R_{E}\right)}{h_{n}^{\prime}\left(k R_{E}\right)} h_{n}\left(k R_{E}\right) .
$$

We assume the desired sound fields are given, and they are produced by a virtual point source located at $(2, \pi / 2, \pi / 8)$ (source \#1) and $(2, \pi / 2,5 \pi / 3)$ (source \#2), respectively, with frequency of $1200 \mathrm{~Hz}$. We calculate the loudspeaker weights using (38) by replacing $\left\{\beta_{n m}^{(\ell)}(k)\right\}$ with $\left\{\hat{\beta}_{n m}^{(\ell)}(k)\right\}$, and plot the sound fields reproduced by the circular array controlled by the velocity-assisted method. The plots including the results of both methods are given in Fig. 14 and Fig. 15, where the difference between the reconstructed sound fields and the desired sound fields for both methods are also shown. Note that in this case the target region is a spherical region with radius of $0.042 \mathrm{~m}$, which is same as the size of the EigenMike. 


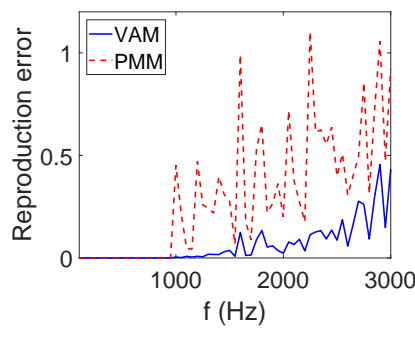

(a)

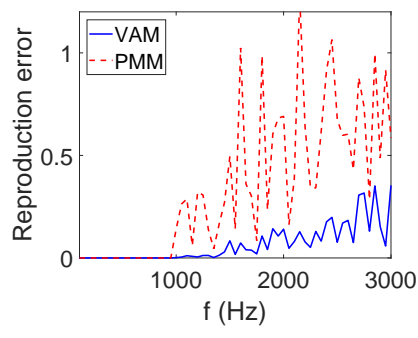

(b)
Fig. 16. 3D reproduction error with respect to the change of frequency controlled by both methods for (a) source \#1 and (b) source \#2.

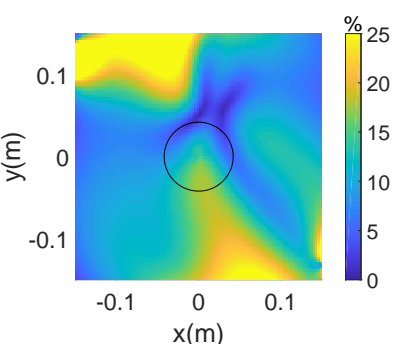

(a)

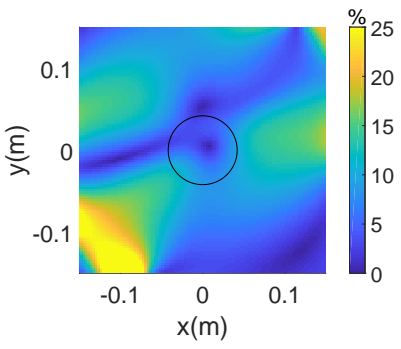

(c)

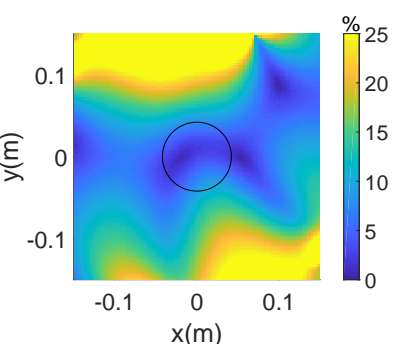

(b)

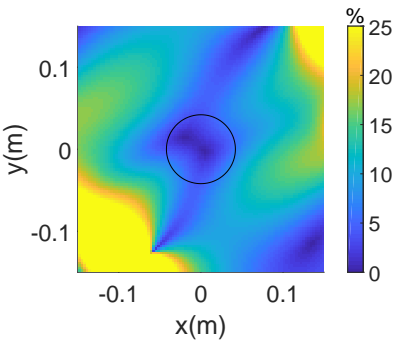

(d)
Fig. 17. The intensity direction error on plane $z=0$ controlled by (a, c) PMM and (b, d) VAM for $(a, b)$ source \#1 and (c, d) source \#2.

With the observation of the error fields between the reproduced and desired pressure fields, the velocity-assisted method can provide accurate pressure reproduction inside the target region, whereas the pressure matching method has around $50 \%$ error for both virtual sources.

The EigenMike can record spatial sound field up to $4^{\text {th }}$ order, therefore, the $\left\{\hat{\beta}_{n m}^{(\ell)}(k)\right\}$ obtained from the measurements is also up to $4^{\text {th }}$ order. Given the radius of $0.042 \mathrm{~m}$, the frequency limit is $3800 \mathrm{~Hz}$ approximately. We process the measurements frequency bin by frequency bin, ranging from $100 \mathrm{~Hz}$ to $3000 \mathrm{~Hz}$. The results showing the reproduction error with respect to different frequency bins for both methods are given in Fig. 16. The six channel loudspeaker array can provide accurate pressure reproduction for the first order system (i.e., when the frequency is less than $950 \mathrm{~Hz}$ ) using both methods. When the frequency goes up, the pressure matching method results in a large error for most of the frequency bins, however, the error of the velocity-assisted method remains low for all the evaluated frequency bins. As shown in Fig. 17, the intensity direction error of the velocity- assisted method within the target region is also less than that of the pressure matching method, which is consistent with the simulation results in Section IV. Consequently, we conclude that the proposed method also has good performance for a real-world environment.

\section{CONCLUSION}

We have proposed a 3D sound field reproduction method based on particle velocity in the modal domain which is appropriate for non-uniform loudspeaker geometries. We derived closed form continuous particle velocity expressions over space based on spherical harmonic coefficients of sound pressure, and designed the driving signals by controlling the continuous particle velocity and sound pressure on the surrounding contour. We evaluated the proposed method in both a free field and a reverberant room by comparing it with the conventional pressure matching in the modal domain. We demostrated that the proposed method can provide more accurate sound field reproduction with a wider frequency range in the target region using a limited number of loudspeakers, as is verified by simulation results. The simulations also revealed that the reflections in the reverberant room can compensate for the non-uniformity of the loudspeaker array and thus improve the reproduction performance. Finally, the results of experiments with the impulse response measurements of a real-world room suggest that the proposed method can also provide good performance in a real-world environment.

\section{APPENDIX A \\ PROOF OF THEOREM 1}

By multiplying both sides of (10) by $Y_{p^{\prime} q^{\prime}}^{*}(\theta, \phi)$ and integrating them with respect to $\theta$ and $\phi$, for the particle velocity in the $\theta$ direction, we have

$$
\begin{aligned}
& \int_{0}^{2 \pi} \int_{0}^{\pi} V_{\theta}(\boldsymbol{x}, k) Y_{p^{\prime} q^{\prime}}^{*}(\theta, \phi) \sin \theta d \theta d \phi= \\
& \int_{0}^{2 \pi} \int_{0}^{\pi} \sum_{p=0}^{\infty} \sum_{q=-p}^{p} X_{p q}^{(\theta)}(k, r) Y_{p q}(\theta, \phi) Y_{p^{\prime} q^{\prime}}^{*}(\theta, \phi) \sin \theta d \theta d \phi
\end{aligned}
$$

Due to the orthogonality of the spherical harmonics, (47) can be simplified as

$$
X_{p q}^{(\theta)}=\int_{0}^{2 \pi} \int_{0}^{\pi} V_{\theta}(\boldsymbol{x}, k) Y_{p q}^{*}(\theta, \phi) \sin \theta d \theta d \phi
$$

Substituting (6) into (48), we have

$$
\begin{aligned}
X_{p q}^{(\theta)} & =\frac{i}{k \rho_{0} c} \int_{0}^{2 \pi} \int_{0}^{\pi} \sum_{n=0}^{N} \sum_{m=-n}^{n} A_{n m} A_{p q} \alpha_{n m}(k) \frac{j_{n}(k r)}{r} \\
& \times P_{n m}^{\prime}(\cos \theta) P_{p q}(\cos \theta) e^{i(m-q) \phi} \sin \theta d \theta d \phi
\end{aligned}
$$

Replacing $P_{n m}^{\prime}(\cos \theta)$ in (49) with (9) and letting $\cos \theta$ be $t$, it reduces to calculate the following two integrals,

$$
\begin{aligned}
\mathcal{P}_{n m p q}^{(\theta)}=\int_{-1}^{1}[ & (n-m+1) \frac{P_{(n+1) m}(t) P_{p q}(t)}{\sqrt{1-t^{2}}} \\
& \left.-(n+1) \frac{t P_{n m}(t) P_{p q}(t)}{\sqrt{1-t^{2}}}\right] d t
\end{aligned}
$$


and

$$
\mathcal{E}_{m q}=\int_{0}^{\pi} e^{i(m-q) \phi} d \phi= \begin{cases}2 \pi, & \text { if } m=q \\ 0, & \text { otherwise. }\end{cases}
$$

To solve the integral of (50), we begin with the transformed Euler integral, adding the product of two hypergeometric polynomials, defined by [54]

$$
\begin{aligned}
\int_{-1}^{1} & \left(1-t^{2}\right)^{\bar{a}-1} t^{2 \bar{b}-1} F\left(-\bar{\mu}_{1}, \bar{\nu}_{1} ; \bar{\xi}_{1} ; 1-t^{2}\right) \\
& \times F\left(-\bar{\mu}_{2}, \bar{\nu}_{2} ; \bar{\xi}_{2} ; 1-t^{2}\right) d t \\
= & \mathcal{G}\left(\bar{a}, \bar{b} ;-\bar{\mu}_{1},-\bar{\mu}_{2} ; \bar{\nu}_{1}, \bar{\nu}_{2}, \bar{\xi}_{1}, \bar{\xi}_{2}\right) \quad(\operatorname{Re}(\bar{a})>0, \operatorname{Re}(\bar{b})>0),
\end{aligned}
$$

where $\operatorname{Re}(\cdot)$ denotes the real part, and

$$
F(-\mu, \nu ; \xi ; x)=\sum_{j=0}^{\mu} \frac{(-\mu)_{j}(\nu)_{j}}{(\xi)_{j} j !} x^{j} .
$$

The associated Legendre function can be represented by the hypergeometric polynomials, which is given as [55]

$$
\begin{aligned}
P_{n m}(t) & =D_{n m}\left[\delta _ { | m | + n } ( 1 - t ^ { 2 } ) ^ { | m | / 2 } F \left(\frac{|m|-n}{2}, \frac{1+|m|}{2}\right.\right. \\
& \left.+\frac{n}{2} ;|m|+1 ; 1-t^{2}\right)+\delta_{|m|+n+1}\left(1-t^{2}\right)^{|m| / 2} t \\
& \left.\times F\left(\frac{1+|m|-n}{2}, \frac{2+|m|+n}{2} ;|m|+1 ; 1-t^{2}\right)\right],
\end{aligned}
$$

where

$$
D_{n m}= \begin{cases}\frac{(-1)^{|m|}(n+|m|) !}{2^{|m|}|m| !(n-|m|) !}, & \text { when } m \geq 0 \\ \frac{1}{2^{|m|}|m| !}, & \text { when } m<0 .\end{cases}
$$

Substituting (54) into (50) together with (52), we have

$$
\begin{aligned}
\mathcal{P}_{n m p q}^{(\theta)} & =(n-m+1) D_{(n+1) m} D_{p q} \mathcal{G}\left(a, b+\frac{1}{2}-\delta_{n+|m|+1} ;\right. \\
& \left.-\mu_{1}-\delta_{n+|m|+1},-\mu_{2} ; \nu_{1}+\delta_{n+|m|}, \nu_{2} ; \xi_{1}, \xi_{2}\right)-(n \\
& +1) D_{n m} D_{p q} \mathcal{G}\left(a, b+\frac{1}{2} ;-\mu_{1},-\mu_{2} ; \nu_{1}, \nu_{2} ; \xi_{1}, \xi_{2}\right) .
\end{aligned}
$$

Substituting (56) and (51) into (49) completes the proof of (12).

Similarly, for the particle velocity in the $\phi$ direction, we have

$$
\begin{aligned}
X_{p q}^{(\phi)} & =-\frac{1}{k \rho_{0} c} \sum_{n=0}^{N} \sum_{m=-n}^{n} m A_{n m} A_{p q} \alpha_{n m}(k) \frac{j_{n}(k r)}{r} \\
& \times \mathcal{P}_{n m p q}^{(\phi)} \mathcal{E}_{m q},
\end{aligned}
$$

where

$$
\begin{aligned}
\mathcal{P}_{n m p q}^{(\phi)} & =\int_{-1}^{1} \frac{P_{n m}(t) P_{p q}(t)}{\sqrt{1-t^{2}}} d t \\
& =D_{n m} D_{p q} \mathcal{G}\left(a, b ;-\mu_{1},-\mu_{2} ; \nu_{1}, \nu_{2} ; \xi_{1}, \xi_{2}\right) .
\end{aligned}
$$

Substituting (58) and (51) into (57) completes the proof of (13).

\section{APPENDIX B \\ PROOF OF EQUATION (29)}

We denote the size of the shoebox room as $\left(L_{x}, L_{y}, L_{z}\right)$ for length, width and height. The room origin $O_{\text {room }}$ is set at the left-front-bottom corner of the room, and the origin $O$ is located at $\boldsymbol{x}_{q o}=\left(x_{q o}, y_{q o}, z_{q o}\right)=\left(L_{x} / 2, L_{y} / 2, L_{z} / 2\right)$ with respect to $O_{\text {room}}$. Therefore, the $\ell^{\text {th }}$ loudspeaker is located at $\boldsymbol{x}_{\ell o}=\boldsymbol{x}_{\ell}+\boldsymbol{x}_{q o}$ with respect to $O_{\text {room. }}$. Reverberant characteristics are modeled with the reflection coefficients of the wall surface, denoted as $\boldsymbol{d}=\left(d_{x 1}, d_{x 2}, d_{y 1}, d_{y 2}, d_{z 1}, d_{z 2}\right)$. According to the spherical harmonics based generalized image source method [46], the point-to-region transfer function in the reverberant environment is given by

$$
\begin{array}{r}
H_{\ell}^{\mathrm{rvb}}(\boldsymbol{x}, k)=\sum_{n=0}^{N} \sum_{m=-n}^{n} \sum_{v=0}^{V} \sum_{u=-v}^{v} \gamma_{v u}^{(\ell)}(k) \alpha_{n m}^{v u}(k) \\
\times j_{n}(k r) Y_{n m}(\theta, \phi),
\end{array}
$$

where $V=\left\lceil k e R_{s} / 2\right\rceil$ is the source region truncation order, $R_{s}$ is the radius of the source region, $\gamma_{v u}^{(\ell)}(k)=$ $i k j_{v}\left(k R_{s}\right) Y_{v u}^{*}\left(\theta_{s}, \phi_{s}\right)$ are the outgoing sound field coefficients due to the $\ell^{\text {th }}$ loudspeaker at $\boldsymbol{x}_{s}=\left(R_{s}, \theta_{s}, \phi_{s}\right)$ with respect to the origin of the source region $O_{s}$, and $\alpha_{n m}^{v u}(k)$ are coupling coefficients, defined by [56]

$$
\begin{aligned}
\alpha_{n m}^{v u}(k) & =\sum_{\boldsymbol{p}=0}^{1} \sum_{\boldsymbol{r}=-\infty}^{\infty} d_{x 1}^{\left|r_{1}-p_{1}\right|} d_{x 2}^{\left|r_{1}\right|} d_{y 1}^{\left|r_{2}-p_{2}\right|} d_{y 2}^{\left|r_{2}\right|} d_{z 1}^{\left|r_{3}-p_{3}\right|} d_{z 2}^{\left|r_{3}\right|} \\
& \times(-1)^{\left(p_{2}+p_{3}\right) u+p_{3} v} S_{v n}^{\left((-1)^{p_{1}+p_{2}} u\right) m}\left(\boldsymbol{x}_{\boldsymbol{p}}+\boldsymbol{x}_{\boldsymbol{r}}\right),
\end{aligned}
$$

where

$$
\begin{aligned}
& S_{v n}^{u m}(\boldsymbol{y})=4 \pi i^{n-v} \\
& \times \sum_{l=0}^{v+n} i^{l}(-1)^{2 u-m} h_{l}\left(k r_{y}\right) Y_{l(m-u)}^{*}\left(\theta_{y}, \phi_{y}\right) W_{1} W_{2} \zeta,
\end{aligned}
$$

$W_{1}=\left(\begin{array}{ccc}v & n & l \\ 0 & 0 & 0\end{array}\right), W_{2}=\left(\begin{array}{ccc}v & n & l \\ u & -m & m-u\end{array}\right)$ denote Wigner $3 \mathrm{j}$ symbols, $\zeta=\sqrt{(2 v+1)(2 n+1)(2 l+1) / 4 \pi}$, $\boldsymbol{x}_{\boldsymbol{p}}=\left(x_{q o}-x_{\ell o}+2 p_{1} x_{\ell o}, y_{q o}-y_{\ell o}+2 p_{2} y_{\ell o}, z_{q o}-z_{\ell o}+\right.$ $\left.2 p_{3} z_{\ell_{0}}\right), \boldsymbol{x}_{\boldsymbol{r}}=\left(2 r_{1} L_{x}, 2 r_{2} L_{y}, 2 r_{3} L_{z}\right), \sum_{\boldsymbol{p}=0}^{1}$ for $\boldsymbol{p}=$ $\left(p_{1}, p_{2}, p_{3}\right)$ and $\sum_{\boldsymbol{r}=-\infty}^{\infty}$ for $\boldsymbol{r}=\left(r_{1}, r_{2}, r_{3}\right)$ both represent triplet summations for indexing room images over three dimensional space. The infinite image depth $r$ is truncated to $\boldsymbol{R}_{\text {depth }}$ for simplicity.

We assume that each loudspeaker constitute a source region and the location of the source coincides with the origin the source region (e.g., $\boldsymbol{x}_{s}=(0,0,0)$ for the $\ell^{\text {th }}$ loudspeaker). Therefore, we have $V=0$, and (59) can be rewritten as (29), which completes the proof.

\section{APPENDIX C PROOF OF EQUATION (40)}

We begin with the following integral

$$
I_{a}(k)=\int_{0}^{R} \int_{0}^{2 \pi} \int_{0}^{\pi}\left|P_{d}(\boldsymbol{x}, k)-P_{a}(\boldsymbol{x}, k)\right|^{2} d \Omega
$$


Substituting (31) and (33) into (62), we have

$$
\begin{aligned}
& I_{a}(k)=\sum_{n=0}^{N} \sum_{m=-n}^{n} \sum_{n^{\prime}=0}^{N} \sum_{m^{\prime}=-n^{\prime}}^{n^{\prime}}\left[\alpha_{n m}^{(d)}(k)-\sum_{\ell=1}^{L} w_{l} \beta_{n m}^{(\ell)}(k)\right] \\
& \times\left[\alpha_{n^{\prime} m^{\prime}}^{(d)}(k)-\sum_{\ell=1}^{L} w_{l} \beta_{n^{\prime} m^{\prime}}^{(\ell)}(k)\right]^{*} \int_{0}^{R} j_{n}(k r) j_{n^{\prime}}(k r) r^{2} d r \\
& \times \int_{0}^{2 \pi} \int_{0}^{\pi} Y_{n m}(\theta, \phi) Y_{n^{\prime} m^{\prime}}(\theta, \phi) \sin \theta d \theta d \phi .
\end{aligned}
$$

According to the orthogonality of the spherical harmonics, it becomes

$$
\begin{aligned}
I_{a}(k) & =\sum_{n=0}^{N} \sum_{m=-n}^{n}\left|\alpha_{n m}^{(d)}(k)-\sum_{\ell=1}^{L} w_{\ell} \beta_{n m}^{(\ell)}(k)\right|^{2} \\
& \times \int_{0}^{R} j_{n}^{2}(k r) r^{2} d r .
\end{aligned}
$$

Also, the integral in term of $r$ is given by [57]

$$
\int_{0}^{R} j_{n}^{2}(k r) r^{2} d r=\mathcal{J}_{n}(k, R)
$$

Therefore, we have

$$
I_{a}(k)=\sum_{n=0}^{N} \sum_{m=-n}^{n}\left|\alpha_{n m}^{(d)}(k)-\sum_{\ell=1}^{L} w_{l} \beta_{n m}^{(\ell)}(k)\right|^{2} \mathcal{J}_{n}(k, R) .
$$

Similarly,

$$
\begin{aligned}
I_{d}(k) & =\int_{0}^{R} \int_{0}^{2 \pi} \int_{0}^{\pi}\left|P_{d}(\boldsymbol{x}, k)\right|^{2} d \Omega \\
& =\sum_{n=0}^{N} \sum_{m=-n}^{n}\left|\alpha_{n m}^{(d)}(k)\right|^{2} \mathcal{J}_{n}(k, R) .
\end{aligned}
$$

Substituting (66) and (67) into (39) completes the proof.

\section{REFERENCES}

[1] A. J. Berkhout, "A holographic approach to acoustic control," J. Audio Eng. Soc., vol. 36, no. 12, pp. 977-995, Dec. 1988.

[2] A. J. Berkhout, D. de Vries, and P. Vogel, "Acoustic control by wave field synthesis," J. Acoust. Soc. Amer., vol. 93, no. 5, pp. 2764-2778, Jan. 1993.

[3] S. Spors, R. Rabenstein, and J. Ahrens, "The theory of wave field synthesis revisited," in Proc. 124th Audio Eng. Soc. Conv., 2008, pp. 17-20.

[4] R. Rabenstein and S. Spors, "Spatial aliasing artifacts produced by linear and circular loudspeaker arrays used for wave field synthesis," in Proc. 120th Audio Eng. Soc. Conv., 2006.

[5] P.-A. Gauthier and A. Berry, "Adaptive wave field synthesis with independent radiation mode control for active sound field reproduction: Theory," J. Acoust. Soc. Amer., vol. 119, no. 5, pp. 2721-2737, Apr. 2006.

[6] M. M. Boone, E. N. Verheijen, and P. F. Van Tol, "Spatial sound-field reproduction by wave-field synthesis," J. Audio Eng. Soc., vol. 43, no. 12, pp. 1003-1012, Dec. 1995.

[7] D. De Vries and M. M. Boone, "Wave field synthesis and analysis using array technology," in Proc. IEEE Workshop Appl. Signal Process. Audio Acoust. (WASPAA), 1999, pp. 15-18.

[8] M. M. Boone, "Multi-actuator panels (maps) as loudspeaker arrays for wave field synthesis," J. Audio Eng. Soc., vol. 52, no. 7/8, pp. 712-723, Jul. 2004.

[9] J. Ahrens and S. Spors, "Sound field reproduction using planar and linear arrays of loudspeakers," IEEE Trans. Audio, Speech, Lang. Process. vol. 18 , no. 8, pp. 2038-2050, Feb. 2010.
[10] S. Spors and J. Ahrens, "Analysis and improvement of pre-equalization in 2.5-dimensional wave field synthesis," in Proc. 128th Audio Eng. Soc. Conv., 2010.

[11] G. Firtha, P. Fiala, F. Schultz, and S. Spors, "Improved referencing schemes for $2.5 \mathrm{~d}$ wave field synthesis driving functions," IEEE/ACM Trans. Audio, Speech, Lang. Process., vol. 25, no. 5, pp. 1117-1127, May 2017.

[12] N. Epain and E. Friot, "Active control of sound inside a sphere via control of the acoustic pressure at the boundary surface," J. Sound Vib. vol. 299, no. 3, pp. 587-604, Jan. 2007.

[13] M. Naoe, T. Kimura, Y. Yamakata, and M. Katsumoto, "Performance evaluation of $3 \mathrm{~d}$ sound field reproduction system using a few loudspeakers and wave field synthesis," in Proc. 2nd Int. Symp. Universal Commun., 2008, pp. 36-41.

[14] F. Fazi, P. Nelson, J. E. Christensen, and J. Seo, "Surround system based on three-dimensional sound field reconstruction," in Proc. 125th Audio Eng. Soc. Conv., 2008.

[15] F. Olivieri, F. M. Fazi, S. Fontana, D. Menzies, and P. A. Nelson, "Generation of private sound with a circular loudspeaker array and the weighted pressure matching method," IEEE/ACM Trans. Audio, Speech, Lang. Process., vol. 25, no. 8, pp. 1579-1591, May 2017.

[16] Y. Cai, M. Wu, and J. Yang, "Sound reproduction in personal audio systems using the least-squares approach with acoustic contrast control constraint," J. Acoust. Soc. Amer, vol. 135, no. 2, pp. 734-741, Feb. 2014.

[17] S. Koyama, K. Furuya, Y. Hiwasaki, and Y. Haneda, "Analytical approach to wave field reconstruction filtering in spatio-temporal frequency domain," IEEE Trans. Audio, Speech, Lang. Process., vol. 21, no. 4, pp. 685-696, Nov. 2012.

[18] Q. Zhu, P. Coleman, X. Qiu, M. Wu, J. Yang, and I. Burnett, "Robust personal audio geometry optimization in the svd-based modal domain,' IEEE/ACM Trans. Audio, Speech, Lang. Process., vol. 27, no. 3, pp. 610-620, Dec. 2018.

[19] G. N. Lilis, D. Angelosante, and G. B. Giannakis, "Sound field reproduction using the lasso," IEEE Trans. Audio, Speech, Lang. Process., vol. 18, no. 8, pp. 1902-1912, Apr. 2010.

[20] N. Radmanesh and I. S. Burnett, "Generation of isolated wideband sound fields using a combined two-stage lasso-ls algorithm," IEEE Trans. Audio, Speech, Lang. Process., vol. 21, no. 2, pp. 378-387, Nov. 2012.

[21] D. B. Ward and T. D. Abhayapala, "Reproduction of a plane-wave sound field using an array of loudspeakers," IEEE Trans. Speech Audio Process., vol. 9, no. 6, pp. 697-707, Sept. 2001.

[22] T. Betlehem and T. D. Abhayapala, "Theory and design of sound field reproduction in reverberant rooms," J. Acoust. Soc. Amer., vol. 117, no. 4 , pp. 2100-2111, Jan. 2005.

[23] Z. Li, R. Duraiswami, and N. A. Gumerov, "Capture and recreation of higher order 3d sound fields via reciprocity," in Proc. Int. Conf. Audio Display, 2004, pp. 6-9.

[24] M. A. Poletti, "Three-dimensional surround sound systems based on spherical harmonics," J. Audio Eng. Soc., vol. 53, no. 11, pp. 1004 1025, Nov. 2005.

[25] J. Ahrens and S. Spors, "Analytical driving functions for higher order ambisonics," in Proc. IEEE Int. Conf. Acoust., Speech, Signal Process. (ICASSP), 2008, pp. 373-376.

[26] M. A. Gerzon, "Ambisonics in multichannel broadcasting and video," $J$. Audio Eng. Soc., vol. 33, no. 11, pp. 859-871, Nov. 1985.

[27] Y. J. Wu and T. D. Abhayapala, "Spatial multizone soundfield reproduction: Theory and design," IEEE Trans. Audio, Speech, Lang. Process. vol. 19, no. 6, pp. 1711-1720, Dec. 2010.

[28] W. Jin and W. B. Kleijn, "Theory and design of multizone soundfield reproduction using sparse methods," IEEE/ACM Trans. Audio, Speech, Lang. Process., vol. 23, no. 12, pp. 2343-2355, Dec. 2015.

[29] W. Zhang, T. D. Abhayapala, T. Betlehem, and F. M. Fazi, "Analysis and control of multi-zone sound field reproduction using modal-domain approach," J. Acoust. Soc. Amer., vol. 140, no. 3, pp. 2134-2144, Sept. 2016.

[30] Y. J. Wu and T. D. Abhayapala, "Theory and design of soundfield reproduction using continuous loudspeaker concept," IEEE Trans. Audio, Speech, Lang. Process., vol. 17, no. 1, pp. 107-116, Dec. 2008.

[31] M. Poletti, F. Fazi, and P. Nelson, "Sound-field reproduction systems using fixed-directivity loudspeakers," J. Acoust. Soc. Amer., vol. 127, no. 6, pp. 3590-3601, Apr. 2010.

[32] A. Gupta and T. D. Abhayapala, "Three-dimensional sound field reproduction using multiple circular loudspeaker arrays," IEEE Trans. Audio, Speech, Lang. Process., vol. 19, no. 5, pp. 1149-1159, Oct. 2010. 
[33] W. Zhang and T. D. Abhayapala, "Three dimensional sound field reproduction using multiple circular loudspeaker arrays: functional analysis guided approach," IEEE/ACM Trans. Audio, Speech, Lang. Process., vol. 22, no. 7, pp. 1184-1194, May 2014.

[34] P. Chen, P. N. Samarasinghe, and T. D. Abhayapala, "3d exterior soundfield reproduction using a planar loudspeaker array," in Proc. IEEE Int. Conf. Acoust., Speech, Signal Process. (ICASSP), 2018, pp. 471475.

[35] M. Poletti, "Robust two-dimensional surround sound reproduction for nonuniform loudspeaker layouts," J. Audio Eng. Soc., vol. 55, no. 7/8, pp. 598-610, Jul. 2007.

[36] N. Ueno, S. Koyama, and H. Saruwatari, "Sound field reproduction with exterior cancellation using analytical weighting of harmonic coefficients," in Proc. IEEE Int. Conf. Acoust., Speech, Signal Process. (ICASSP), 2018, pp. 466-470.

[37] J. Zhang, W. Zhang, T. D. Abhayapala, and L. Zhang, " 2.5 d multizone reproduction using weighted mode matching: Performance analysis and experimental validation," J. Acoust. Soc. Amer, vol. 147, no. 3, pp. 1404-1417, Feb. 2020.

[38] N. Ueno, S. Koyama, and H. Saruwatari, "Three-dimensional sound field reproduction based on weighted mode-matching method," IEEE/ACM Trans. Audio, Speech, Lang. Process., vol. 27, no. 12, pp. 1852-1867, Aug. 2019.

[39] W. Jin, "Adaptive reverberation cancelation for multizone soundfield reproduction using sparse methods," in Proc. IEEE Int. Conf. Acoust., Speech, Signal Process. (ICASSP), 2016, pp. 509-513.

[40] P. Setiawan and W. Jin, "Compressing higher order ambisonics of a multizone soundfield," in Proc. IEEE Int. Conf. Acoust., Speech, Signal Process. (ICASSP), 2017, pp. 466-470.

[41] M. Shin, F. M. Fazi, P. A. Nelson, and J. Seo, "Control of velocity for sound field reproduction," in Proc. 52th Int. Audio Eng. Soc. Conf., 2013.

[42] M. Shin, P. A. Nelson, F. M. Fazi, and J. Seo, "Velocity controlled sound field reproduction by non-uniformly spaced loudspeakers," J. Sound Vib., vol. 370, Feb. 2016

[43] M. Buerger, R. Maas, H. W. Löllmann, and W. Kellermann, "Multizone sound field synthesis based on the joint optimization of the sound pressure and particle velocity vector on closed contours," in Proc. IEEE Workshop Appl. Signal Process. Audio Acoust. (WASPAA), 2015, pp. $1-5$.

[44] R. A. Kennedy, P. Sadeghi, T. D. Abhayapala, and H. M. Jones, "Intrinsic limits of dimensionality and richness in random multipath fields," IEEE Trans. Signal Process., vol. 55, no. 6, pp. 2542-2556, Jun. 2007.

[45] E. G. Williams, Fourier acoustics: sound radiation and nearfield acoustical holography. Elsevier, 1999.

[46] P. N. Samarasinghe, T. D. Abhayapala, Y. Lu, H. Chen, and G. Dickins, "Spherical harmonics based generalized image source method for simulating room acoustics," J. Acoust. Soc. Amer, vol. 144, no. 3, pp. 1381-1391, Aug. 2018.

[47] M. Acoustics, "Em32 eigenmike microphone array release notes (v17. 0)," 25 Summit Ave, Summit, NJ 07901, USA, 2013.

[48] C. F. Van Loan and G. H. Golub, Matrix computations. Johns Hopkins University Press, 1983.

[49] N. J. A. Sloane, R. H. Hardin, and W. D. Smith, Spherical Codes. [Online]. Available: http://neilsloane.com/packings/.

[50] K. Hamasaki, K. Hiyama, and R. Okumura, "The 22.2 multichannel sound system and its application," in Proc. 118th Audio Eng. Soc. Conv., 2005.

[51] E. A. Lehmann, A. M. Johansson, and S. Nordholm, "Reverberationtime prediction method for room impulse responses simulated with the image-source model," in Proc. IEEE Workshop Appl. Signal Process. Audio Acoust. (WASPAA), 2007, pp. 159-162.

[52] M. A. Gerzon, "General metatheory of auditory localisation," in Proc. 92nd Audio Eng. Soc. Conv., 1992.

[53] M. Frank, Phantom sources using multiple loudspeakers in the horizontal plane. Ph.D. dissertation, Univ. Music and Performing Arts Graz, Austria, 2013.

[54] H. Zuo, P. N. Samarasinghe, T. D. Abhayapala, and G. Dickins, "Spatial sound intensity vectors in spherical harmonic domain," J. Acoust. Soc. Amer., vol. 145, no. 2, pp. EL149-EL155, Jan. 2019.

[55] T. P. Higgins and Z. Kopal, "Volume integrals of the products of spherical harmonics and their application to viscous dissipation phenomena in fluids," Astrophys. Space Sci., vol. 2, no. 3, pp. 352-369, Nov. 1968.

[56] L. I. Birnie, T. D. Abhayapala, and P. Samarasinghe, "Reflection assisted sound source localization through a harmonic domain music framework," IEEE/ACM Trans. Audio, Speech, Lang. Process., Nov. 2019.
[57] J. K. Bloomfield, S. H. Face, and Z. Moss, "Indefinite integrals of spherical bessel functions," arXiv preprint arXiv:1703.06428, 2017.

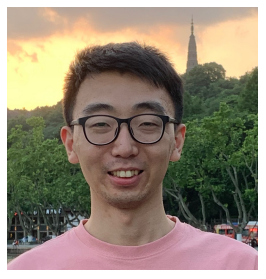

Huanyu Zuo received the Bachelor's degree and Master's degree in mechatronical engineering from the Beijing Institute of Technology, Beijing, China, in 2014 and 2017, respectively. He is currently working towards the Ph.D. degree in audio \& acoustic signal processing at the Australian National University (ANU), Canberra, Australia. His research interests include spatial sound recording and reproduction, 3D sound field separation, and psychoacoustics.

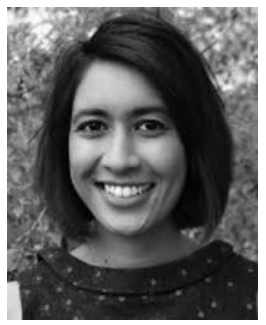

Prasanga N. Samarasinghe received the B.E. (Hons.) degree in electronic and electrical engineering from the University of Peradeniya, Peradeniya, Sri Lanka, in 2009, and the Ph.D. degree from the Australian National University (ANU), Canberra, ACT, Australia, in 2014. She is currently a Research Fellow with the Research School of Electrical, Energy and Materials Engineering, ANU. Her research interests include spatial sound recording and reproduction, spatial noise cancellation, and array optimization using compressive sensing techniques.

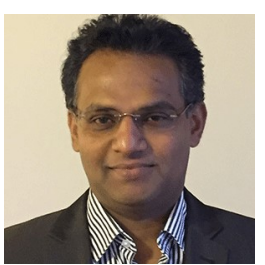

Thushara D. Abhayapala received the B.E. degree in engineering and the Ph.D. degree in telecommunications engineering from Australian National University (ANU), Canberra, ACT, Australia, in 1994 and 1999, respectively. He is a Professor of Signal Processing with ANU, Canberra, ACT, Australia. He held a number of leadership positions including Deputy Dean of the College of Engineering and Computer Science (2015-2019), Head of the Research School of Engineering with ANU (2010-2014) and the Leader of the Wireless Signal Processing Program at the National ICT Australia from 2005-2007. His research interests are in the areas of spatial audio and acoustic signal processing, and multichannel signal processing. Among many contributions, he is one of the first researchers to use spherical harmonic based eigen-decomposition in microphone arrays and to propose the concept of spherical microphone arrays; novel contributions on the multi-zone sound field reproduction problem; was one of the first to show the fundamental limits of spatial sound field reproduction using arrays of loudspeakers and spherical harmonics. He worked in industry for two years, before his doctoral study and has active collaboration with a number of companies. He has supervised $36 \mathrm{Ph} . \mathrm{D}$. students and co-authored more than 280 peer-reviewed papers. He was an Associate Editor for the IEEE/ACM TRANSACTIONS ON AUDIO, SPEECH, AND LANGUAGE PROCESSING and was a member of the Audio and Acoustic Signal Processing Technical Committee (2011-2016) of the IEEE Signal Processing Society. He is a fellow of Engineers Australia (IEAust). 